\title{
A review on process intensification in HiGee distillation
}

DOI:

$10.1002 / j c t b .5206$

\section{Document Version}

Accepted author manuscript

Link to publication record in Manchester Research Explorer

\section{Citation for published version (APA):}

Cortes Garcia, G. E., van der Schaaf, J., \& Kiss, A. A. (2017). A review on process intensification in HiGee distillation. Journal of Chemical Technology and Biotechnology, 92(6), 1136-1156.

https://doi.org/10.1002/jctb.5206

\section{Published in:}

Journal of Chemical Technology and Biotechnology

\section{Citing this paper}

Please note that where the full-text provided on Manchester Research Explorer is the Author Accepted Manuscript or Proof version this may differ from the final Published version. If citing, it is advised that you check and use the publisher's definitive version.

\section{General rights}

Copyright and moral rights for the publications made accessible in the Research Explorer are retained by the authors and/or other copyright owners and it is a condition of accessing publications that users recognise and abide by the legal requirements associated with these rights.

\section{Takedown policy}

If you believe that this document breaches copyright please refer to the University of Manchester's Takedown Procedures [http://man.ac.uk/04Y6Bo] or contact uml.scholarlycommunications@manchester.ac.uk providing relevant details, so we can investigate your claim.

\section{OPEN ACCESS}




\section{A review on process intensification in HiGee distillation}

${ }^{1}$ Laboratory of Chemical Reactor Engineering, Department of Chemical Engineering \&

5 Chemistry, Eindhoven University of Technology, P.O. Box 513, 5600 MB Eindhoven, The

6 Netherlands.

$7 \quad{ }^{2}$ AkzoNobel Research, Development \& Innovation, Process Technology SRG, Zutphenseweg

8 10,7418 AJ Deventer, The Netherlands.E-mail:Tony.Kiss@akzonobel.com

$9{ }^{3}$ Sustainable Process Technology Group, Faculty of Science and Technology, University of Twente, P.O. Box 217, 7500 AE Enschede, The Netherlands

\section{Keywords}

HiGee, distillation, gas-liquid contactor, heterogeneous catalyst, rotating packed bed

\section{Abstract}

This review paper describes the state-of-the-art in the field of HiGee contactors used for gasliquid mass transfer processes, with a special focus on distillation, and for heterogeneously catalyzed reactions.

Several types of rotating beds are discussed, including single-block rotating packed-bed, splitpacking rotating bed, rotating zigzag bed, two-stage counter-current rotating packed bed, blade packing rotating packed bed, rotating bed with blade packing and baffles, counter-flow concentric-ring rotating bed and crossflow concentric-baffle rotating bed.

The working principles of HiGee technology, as well as the modeling, design and control aspects, and practical applications are explained and discussed. In addition, this paper addresses the advantages and disadvantages with respect to mass-transfer performance, pressure drop, rotor complexity and suitability to perform continuous distillation and to be filled with catalyst packing for heterogeneous reactions. 


\section{1. Introduction}

2 The idea of exploiting high-gravity fields to intensify gas-liquid mass transfer has received 3 special attention over the last three decades, after a patent was granted to Ramshaw and 4 Mallinson in $1981^{1}$. However, this concept has been around for more than a century, since Elsenhans filed a patent in $1906^{2}$ for a non rotor-stator rotating zigzag bed for purifying gases. Schmidt patented the first rotating packed bed with wire mesh packing in $1913^{3}$. Then, Placek was granted several patents, between 1933 and 1944, for a spiraling rotating bed ${ }^{4}$, a corrugated plates with holes rotating bed ${ }^{5,6}$ and a concentric rings with holes rotating bed ${ }^{7}$. Thereafter, Kapitza patented the first rotor-stator zigzag bed in $1952^{8}$ and Pilo and Dahlbeck filed several patents ${ }^{9-11}$ during the 60's for rotating packed beds for gas absorption and desorption, distillation and reaction. However, Podbielniak was the first to address the effect of gravity on the tallness of the distillation column in $1935^{12}$ and continued his efforts on process intensification in distillation and extraction for more than two decades ${ }^{13-24}$. HiGee contactors operate in a high-gravity field (100-1000 times gravity) in order to enhance mass-transfer and throughput up to 1-2 orders of magnitude, allowing for a reduction in size of up to 10 times with respect to conventional packed columns for the same separation ${ }^{25-27}$. This size reduction has been attributed to their higher volumetric mass-transfer coefficients and intensified momentum and heat transfer rates due to the formation of thinner liquid films and smaller droplets which result in larger surface areas and improved micromixing performance with respect to conventional packed bed equipment ${ }^{28,29}$, offering the possibility to use very high specific surface area packing. Additionally, high gravity relaxes flooding limits that allow operation at higher gas velocities.

The high-gravity (HiGee) gas-liquid contactor most commonly mentioned in the literature is the single-block rotating packed bed (RPB). However, subsequent developments have resulted in several HiGee contactors with varying hydraulic and mass transfer characteristics, depending mostly on their rotor design. These rotors can be rather complex due to the combination of moving and stationary disks or even two disks moving in counter-direction. The rotating zigzag bed seems to be of the best rotating beds for performing continuous distillation because it allows for intermediate feeds within one single rotor and because of its favorable combination of high mass transfer performance and higher liquid residence time when compared to other rotating beds. However, its rotor is formed by concentric rings or baffles that do not allow for catalyst to be used as packing. An upgraded version of the rotating zigzag bed, the two-stage counter-current rotating packed bed ${ }^{30,31}$, combines the advantages of the rotating zigzag bed with the capability to use packing and therefore is seen, 
1 together with the much simpler conventional $\mathrm{RPB}^{1}$ (two-stage), as the most appropriate

2 equipment to attempt heterogeneous-catalyzed reactions among all HiGee contactors available 3 in the literature ${ }^{30,31}$.

4 A disadvantage of HiGee gas-liquid contactors is their higher pressure drop compared to conventional packed beds, however, the main disadvantage are the rotating parts, including the rotor, bearings and dynamic seals, which result in long-term reliability concerns ${ }^{26}$. This disadvantage is mitigated by the fast and simple shutdown and startup of these equipment ${ }^{32}$, making it easy to perform preventive and corrective maintenance, without incurring high production losses.

Several papers discussing HiGee designs have been published so far, including multi-stage spraying rotating packed bed $^{33}$, RPB with wave-form disk packing ${ }^{34}$, helical rotating absorber $^{35}$, RPB with split packing (SP-RPB) ${ }^{30,36-39}$, rotating zigzag bed (RZB) ${ }^{27,40,41}$, twostage counter-current rotating packed bed (TSCC-RPB) ${ }^{30,31,36}$, blade-packing rotating packedbed $(\mathrm{BP}-\mathrm{RPB})^{42-47}$, counter-flow concentric-ring rotating bed ${ }^{48}$, cross-flow concentric-baffle rotating bed ${ }^{49}$. This review paper provides an overview of the HiGee technology available both for gas-liquid mass-transfer -with a special focus on distillation- and for heterogeneously catalyzed reactions. First, the working principles of different rotating beds reported in the literature are explained. Next, modeling, design and control aspects of HiGee equipment are presented. Finally, a current list of industrial applications is presented and discussed.

\section{Working principles}

The essence of HiGee technology is replacing the gravitational field by a high centrifugal field achieved by rotating a cylindrical rigid bed. Figure 1 illustrates the working principle of HiGee distillation in a rotating packed bed (RPB) with a vertical axis. The vapor-liquid counter-current flow is horizontal in case of HiGee distillation and not vertical, as typical for conventional operation. This means that the separation extent is determined by the diameter of the rotor while its capacity is limited by its axial height - in contrast with conventional distillation where the diameter determines the capacity and the height of the column gives the separation extent ${ }^{50,51}$. The rotor is an annular, cylindrical packed bed, a series of concentric perforated baffles or a combination of both, housed in a casing and driven by a motor. Several different HiGee devices have been developed over the last 30 years and the most relevant ones are described in the next section. They differ mainly in their rotor design, since this determines the main characteristics of rotating beds. 


\section{3. HiGee equipment}

\section{$2 \quad 3.1$ Single-block rotating packed-bed (RPB)}

3 A single-block RPB is formed by a casing containing a rotor filled with packing, a shaft, a

4 liquid distributor and gas and liquid inlet/outlets. The rotor can be made up of diverse porous media, such as wound wire mesh, foam metal, and corrugated sheet metal. The liquid is injected onto the center of the rotor through a stationary set of nozzles (distributor) and is thrown out into the packing. Then, it flows radially outwards as thin films, rivulets, or droplets by centrifugal force and leaves the packing as a shower of droplets, which is collected by the casing wall and runs downwards along the walls by the action of gravity, leaving the casing. The gas, on the other hand, can flow in co-current, counter-current or cross-current, with respect to the liquid; being the counter-current and cross-current arrangements the most commonly used for gas-liquid contacting. These two different gas flow arrangements are described in more detail down below.

Chen et al. ${ }^{52}$ and Yang et al. ${ }^{53}$ demonstrated that the highest mass transfer rates in an RPB take place at the inner-end zone of the rotor $\left(r_{i}\right)$ due to several reasons: Firstly, because the most violent gas-packing collisions occur at this location due to the high relative velocity between the incoming liquid jets and the rotating packing. Secondly, because at $r_{i}$, the crosssectional area for flow is minimum and the gas flux is maximum. And lastly, because the most rapid resupply of fresh liquid from the liquid distributor occur at this place. Counter-current flow rotating packed bed

A simplified drawing of an RPB operating in counter-current flow is shown in Figure 2 (top). The liquid is injected onto the center of the rotor and flows radially outwards as previously described. The gas enters the equipment casing and then the rotor at the outer periphery and is forced to flow radially inwards due to the pressure gradient. The gas and the liquid contact each other counter-currently while mass transfer occurs. The gas leaves the packing at the eye of the rotor through the outlet pipe. ${ }^{27,50}$ A mechanical seal is required in order to block any gas bypass flow around the rotor. ${ }^{54}$ In a counter-current flow rotating packed bed the gas flow rate is limited by the flow area at the eye of the rotor where flooding is most likely to occur since it is where the gas and liquid velocities are highest.

Cross-current flow rotating packed bed

31 A simplified drawing of a cross-current flow RPB is shown in Figure 2 (btm). The liquid is injected onto the center of the rotor and flows radially outwards as previously described. The gas enters at the bottom, flows axially through the packing and leaves the rotor from the top. 
1 Therefore, the gas and the liquid contact each other in a cross-current flow in the rotor. In contrast with the counter-current RPB, in the cross-current flow RPB the gas is not withdrawn from the eye of the rotor and therefore the flooding constraint can be relaxed, enabling its operation at higher gas flow rates ${ }^{55,56}$. For instance, Guo et al. ${ }^{56}$ studied the hydrodynamics and mass transfer characteristics in a cross-current flow rotating packed bed and found that it could operate at gas flow rates as high as $15 \mathrm{~m} / \mathrm{s}$ without flooding. At such high gas flow rates, the droplets formed at the top of the packing are likely to be instantaneously entrained by the gas. This can prevented by having a section of non-irrigated packing at the downstream side to separate out the droplets by inertial impact ${ }^{56}$. The cross-current flow arrangement has been reported to result in larger volumetric mass transfer coefficients than counter-current flow RPBs but with a lower pressure drop at the same operational conditions ${ }^{57,58}$.

Both counter-current and cross-current flow single-block RPBs have some drawbacks. The liquid residence time inside the rotor is very short, which limits the separation extent despite the very high mass transfer rates ${ }^{40}$. Since the whole packing is rotating, it is difficult to insert middle-feed streams and therefore, multiple rotors are required for continuous distillation. This is not, however, a crucial downside since the limited amount of equilibrium stages available per rotor makes multiple rotors necessary for distillation anyway. A coaxial multirotor configuration (i.e. multiple rotors coaxially installed in one casing) is structurally complicated due to the need of dynamic seals and liquid collectors ${ }^{40}$ and for this reason, multirotor configurations usually involve the use of two or three rotating packed beds driven by individual motors. In addition to that, Rao et al. ${ }^{25}$ and Sandilya et al. ${ }^{59}$ showed that although both volumetric mass transfer coefficients $\left(\mathrm{k}_{\mathrm{G}} \mathrm{a}\right.$ and $\left.\mathrm{k}_{\mathrm{L}} \mathrm{a}\right)$ increase with the rotational speed, this is due to larger interfacial areas (a), to enhanced liquid-side mass transfer coefficients $\left(\mathrm{k}_{\mathrm{L}}\right)$ and to the intense mass transfer in the entry region (end effect ${ }^{52,53}$ ), but not to enhancements in the gas-side mass transfer coefficients $\left(\mathrm{k}_{\mathrm{G}}\right)$, which are only marginal. They suggest that this occurs because the gas acquires the same angular velocity of the packing soon as it enters the rotor and starts rotating as a rigid body along with the packing. Sandilya et al. ${ }^{59}$ compared experimental results of $\mathrm{k}_{\mathrm{G}}$ in an RPB with those calculated using the correlation proposed by Onda et al. ${ }^{60}$ for conventional packed columns, given by

$$
k_{G}=C\left(\frac{D_{G} a_{t}}{R T}\right) R e_{G}^{0.7} S c_{G}^{1 / 3}\left(a_{t} d_{p}\right)^{-2}
$$

They found that the $\mathrm{k}_{\mathrm{G}}$ values in the RPB were even lower than the ones estimated using Onda's correlation and attributed this to liquid maldistribution. Thus the gas flow is similar to 
1 that through a stationary rotor, the gas-side mass transfer coefficients lie in a similar range 2 than for conventional trickle beds and no intensification of the gas-side mass transfer is expected other than that due to the larger interfacial area. The gas velocity, however, can increase significantly compared to trickle beds under the high-gravity field, and, according to equation (1), this leads to larger gas-side mass transfer coefficients since $\mathrm{k}_{\mathrm{G}}$ depends on the gas Reynolds number.

\subsection{Rotating packed-bed with split packing (SP-RPB)}

Chandra et al. ${ }^{61}$ came up with an alternative rotating packed bed design (Figure 3 ) in which the rotor was split into two sets of alternate annular rings of packing (wire mesh or metal foam) with gaps in between. One of the sets was fixed to the top disk and the other one to the bottom disk. The two sets of rings were rotated with two motors in a counter- or co-direction to promote gas tangential slip inside the packing and increase the gas-side mass transfer coefficient, as suggested by Rao et al. ${ }^{59}$.

The SP-RPB design is much more complex than the single-block RPB, especially due to the need for two rotors spinning independently ${ }^{39}$. In spite of this, recent studies ${ }^{39}$ have suggested that the split-packing RPB design may be superior over conventional single-block RPB only for gas-side resistance controlled mass transfer processes. Similar to the single-block RPB, it is difficult to insert middle-feed streams in a SP-RPB since both the upper and lower disks are rotating during operation ${ }^{61,62}$. Additionally, a multistage configuration of rotor-rotor stages on a single axis is mechanically impossible.

\subsection{Rotating zigzag bed (RZB)}

In 1952, Kapitza $^{8}$ patented the so-called rotating zigzag bed. Later, in 2008 , Ji et al. ${ }^{63}$ patented the rotating zigzag bed ${ }^{40,41}$ again. The RZB is composed of a rotating and a stationary disk as shown in Figure 4. In the rotating zigzag bed, concentric circular baffles are fixed on the rotational and stationary disks and serve as the contacting elements of gas and liquid phases. These baffles are assembled together, alternating between rotational and stationary baffles. The rotational baffles have perforations on their upper part and are fixed on the lower (rotational) disk. The stationary baffles are fixed on the upper disk. The gas and liquid flow in zigzag through the clearance between the rotational baffles and the upper disk and through the clearance between stationary baffles and the lower disk. As in the single-block RPB, the gas is fed through the casing and is forced to flow to the rotor and radially inwards through the 
1 packing due to the pressure gradient. The liquid is fed at the center of the rotor and flows

2 radially outwards, contacting the gas in counter-current, due to the centrifugal force.

3 In a RZB the liquid is thrown by centrifugal force from the rotational baffles into the

4 stationary baffles, resulting in very fine droplets. For this reason, the rotating zigzag bed can

5 function without liquid distributors ${ }^{27,40,41}$. In addition to that and thanks to its upper disk being stationary, the dynamic seal can be eliminated and intermediate feeds can be easily introduced at any radial length, making continuous distillation possible without the need of two rotors, provided that the required number of theoretical stages can be reached within a single rotor. The mass transfer performance of a rotating zigzag bed is comparable to that of a single-block rotating packed bed but with better operability at a higher turndown ratio ${ }^{40}$ (ratio of the highest and lowest achievable flow rates). However, its separation ability can be further improved by simply installing multiple rotors in one casing. The RZB, on the other hand, has a higher power consumption than the single-block RPB since every time the liquid contacts the static baffles it is brought to a halt and must be accelerated again by the rotating baffles. Additionally, the zigzag path of the gas results in a higher gas pressure drop compared to an $\mathrm{RPB}^{40,41,64}$. The fact that no packing is used in the RZB, significantly reduces its available surface area for gas-liquid contacting ${ }^{30,40,41}$, making it unsuitable for heterogeneously catalyzed reactions.

\subsection{Two-stage counter-current rotating-packed bed (TSCC-RPB)}

Luo et al. ${ }^{30}$ developed and investigated a design that combined the packed bed and the zigzag rotor design. The TSCC-RPB (Figure 5) has two stages and each stage has a rotor ${ }^{31}$. The two rotors are installed on one shaft driven by one motor and each rotor is made up of a rotating disk, fixed to the shaft, and a stationary disk, fixed to the housing. Packing rings and concentric rings are attached to the rotating disk and the stationary disk, respectively, to enhance the collision between the liquid and the packing and to lengthen the contact time. Conventional packing or catalysts can be loaded into the concentric rotating rings for distillation, heterogeneously catalyzed reactions and/or catalytic (homogenous and heterogeneous) reactive distillation ${ }^{30}$.

30 The liquid is fed into the upper rotor through a stationary distributor, and it moves outwards due to the centrifugal force, passing through both the porous stationary rings and packed rotating rings. The liquid is then collected at the bottom of the upper housing and flows into the eye at the center of the lower rotor. It then flows into the lower rotor and radially outwards, leaving the RPB from the liquid outlet. 
1 The gas is tangentially introduced into the TSCC-RPB from the gas inlet and flows in sequence through the lower rotor, the eye of the lower rotor and the upper rotor before leaving the rotating packed-bed through the gas outlet. The liquid and the gas are thus contacted counter-currently while mass transfer takes place.

Since the TSCC-RPB has two stages, it can be used for continuous distillation, with the upper rotor serving as a rectifying section and the lower rotor as a stripping section. Besides, intermediate feeds can also be introduced by installing liquid distributors on top of the stationary disk, making continuous distillation possible with a single rotor, provided the number of equilibrium stages per rotor is enough for the required separation.

The main drawback of the TSCC-RPB is its complex rotor structure with the combination of rotational packing and static rings that require high manufacture precision, resulting in high costs that brings additional to its industrial application ${ }^{36}$.

\subsection{Blade packing-rotating packed-bed (BP-RPB)}

Lin et al. ${ }^{42}$ developed an RPB equipped with blade packings in order to achieve a low gas pressure drop compared to previous HiGee contactors. The packings were made up of 12 blades covered in stainless steel wire mesh that were installed inside the rotor, keeping a separation angle of 30 degrees among each other (Figure 6). Liquid left the distributor at a relatively high velocity and then entered the inner side of the rotor and moved radially outwards while contacting the gas counter-currently. However, the structure of the rotor with blades is limited by the number of blades and therefore has a lower surface area compared to a conventional rotating packed bed ${ }^{65}$.

Other variations of HiGee gas-liquid contactors combining packing and blades ${ }^{44}$, and blade packing and baffles ${ }^{45}$ have been also developed. Luo et al. ${ }^{44}$ came up with a rotor with packing and blades shown in Figure 7. This rather complex rotor was developed based on previous observations about the end effect in $\operatorname{RPBs}^{52,53}$. In their work, Luo et al. ${ }^{44}$ built and tested five different rotors equipped with packing and blades to artificially create multiple end zones inside the rotors and thus enhance mass transfer in the so-called bulk zone (the zone of an RPB rotor where the end effect is not dominant). These rotors had three rings of packing sections separated by two rings of blades. The design parameter changed among the five rotors was the angle between the plane of their blades and the equatorial line of the rotor (Figure 7). The experimental results reported ${ }^{44}$ indicate that the rotors with packing and blades can intensify the mass transfer process over a range of gas-liquid ratios, resulting in both larger mass transfer coefficients and specific surface areas than single-block rotating 
1 packed beds. They attributed this to the disintegration of the liquid into tiny liquid droplets caused by the more energetic gas-liquid interactions in the so-called "artificially-created end zones" between the high-voidage packing and the blades. The main drawback of this design is the higher complexity of its rotor structure compared to a conventional RPB rotor ${ }^{44}$. Sung and $\mathrm{Chen}^{45}$ came up with another variation of the blade-packing rotating packed-bed which has a rotor with blade packings and static baffles. A schematic drawing of this rotor is shown in Figure 8 . The blade packings and stationary baffles are alternately aligned in the radial direction of the rotor and are fixed to a rotational and a stationary disk, respectively. The stationary baffles were fixed at the clearance between sets of blade packings keeping a distance of $2 \mathrm{~cm}$ between the two disks. These baffles retard gas rotation and provide a high annular slip velocity between the gas and packing while producing a lower pressure drop than in an RPB and a rotating bed with blade packings ${ }^{45}$.

\subsection{Counter-flow concentric-ring rotating bed}

The rotor of the counter-flow concentric-ring rotating bed, CFCR-RPB (Figure 9), was developed by $\mathrm{Li}$ et al. ${ }^{48}$ in an attempt to improve the RZB by perforating all parts of the rotating baffles (not only the top, as in the RZB) and by eliminating the stationary baffles. The rotor comprises a rotating disk, driven by a motor, and a stationary disk. A set of concentric circular metal rings with small perforations acting as gas and liquid channels is fixed to the rotating disk with equal radial spacing. Concentric circular grooves are made on the lower surface of the stationary disk such that when the two disks are assembled, the top of the concentric rotating rings extends into the concentric grooves, forming a tight labyrinth seal to prevent gas from bypassing the rotating rings. Intermediate feeds can be introduced on top of the stationary disk, which is attached to the casing.

The liquid enters through the liquid inlet of the rotating bed and flows to the rotating liquid distributor, where it is dispersed as fine liquid droplets into the center of the bed. The liquid droplets flow radially outwards through the perforations on the rotating rings due to the centrifugal force and they are discharged through the liquid outlet of the casing. The gas is tangentially introduced into the casing and flows radially inwards through the perforations of rotating rings due to the pressure difference and is discharged through the gas outlet. Gas and liquid contact each other counter-currently while mass transfer takes place.

Li et al. ${ }^{48}$ performed total reflux distillation experiments at atmospheric pressure using an ethanol-water system in a counter-flow concentric-ring rotating bed. They compared the equipment performance with that of an RZB and found that even though the counter-flow 
concentric-ring rotating bed had a lower mass transfer performance, its gas-liquid throughput was at least 5.6 times greater than that of RZB, which reached its flooding limits at an Ffactor of $0.66 \mathrm{~m} / \mathrm{s}\left(\mathrm{kg} / \mathrm{m}^{3}\right)^{0.5}$ while the counter-flow concentric ring rotating bed could operate without flooding up to an F-factor of $3.68 \mathrm{~m} / \mathrm{s}\left(\mathrm{kg} / \mathrm{m}^{3}\right)^{0.5}$. Additionally, its pressure drop per discrete step was comparable to that of the RZB at different F-factors until the flooding limit of the RZB was reached and suddenly increased its pressure drop.

\subsection{Cross-flow concentric-baffle rotating bed (CRB)}

Another modified version of the RZB is the crossflow concentric-baffle rotating bed (CRB) developed by Wang et al. ${ }^{49}$ (Figure 10). In the CRB the gas flows in zigzag towards the center of the bed while the liquid flows radially outwards, contacting the gas in cross-current flow.

The rotor comprises a set of perforated concentric baffles, which are fixed to the rotational disk and extend into concentric grooves made into the lower surface of the stationary disk, as described previously for the counter-flow concentric ring rotating bed. Each baffle is divided into three zones in the axial direction: gas-hole zone, liquid-hole zone, and non-hole zone. The baffles are fixed to the rotational disk in such a way that alternate baffles are in axially opposite directions. Due to pressure difference, gas flows through the zigzag flow channels formed by the gas-hole zones on all baffles and the annular space between the adjacent baffles. Liquid flows radially outwards due to the centrifugal force, passing as fine droplets through the liquid-hole zones on all baffles. The liquid is then collected on the casing wall and leaves the rotating bed through the liquid outlet.

The crossflow concentric-baffle rotating bed has lower shaft power requirements and little backmixing compared to the rotating zigzag bed. However, the stage efficiency of the CRB is one-third as much as that of the RZB, or even lower. The stage efficiency is here defined as the ratio between the number of theoretical stages achieved in the rotating bed and the number of contacting stages (annular spaces between adjacent rotational baffles).

\subsection{Other HiGee equipment}

\section{Multi-stage spraying rotating packed bed}

A multi-staged spraying rotating bed is similar to the crossflow RPB in flow mode, the rotor of which comprises of multiple concentric packing rings. In the "spraying zone", the liquid was sprayed into fine droplets with a large interfacial area due to centrifugal force. Its disadvantage is potential liquid entrained by gas flow despite lower gas frictional resistance. ${ }^{40}$ 
Rotating packed-bed with wave-form disk packing

2 To effectively reduce gas flow resistance, a waveform disk rotating bed was developed, in

3 which the rotor contains a series of concentric waveform disks. This rotating bed can utilize

4 the extended interfacial area generated by atomization besides the surface area of disk. ${ }^{40}$

$5 \quad$ Helical rotating bed

6 A helical rotating bed features four spiral blades installed on a rotating disk. Due to the small contact area, its mass transfer performance is still lower compared to a rotating packing bed in spite of the longer flow channel and residence time of gas and liquid. For a helical rotating bed, long liquid residence time in the rotor is offset by the small interfacial area, which results in low volumetric mass transfer coefficients. ${ }^{40}$

Table 1 shows a list of the gas-liquid HiGee contactors that have been described in this section, along with a summary of their advantages and disadvantages, as well as their applications reported in the open literature. Table 2 summarizes the results of relevant experimental studies of HiGee distillation reported in the literature.

It should be noted that, contrary to what is expected, the superficial gas and liquid velocities often employed in HiGee are only a fraction of those used in packed columns. Such flow rates lead to very low HTU and HETP values, but they may not be useful for design. Overall, the HETP values shown in Table 2 vary widely between $1.02 \mathrm{~cm}$ and $15.0 \mathrm{~cm}$ and their variation depends on the rotor design, the separation system and the operating conditions during the experiments. However, the incomplete or no specification of the liquid distributor and/or the packings in many HiGee studies, added to the aforementioned low superficial velocities offsets the usefulness of the mass transfer data reported and makes it difficult to fairly compare different HiGee configurations. For instance, the $\mathrm{K}_{\mathrm{G}} \mathrm{a}$ data reported by Nascimento et al. $^{66}$ is several orders of magnitude higher than the data reported by Ramshaw and Mallinson ${ }^{1}$. Since the former does not report gas flows, it is not possible to explain this differences based on gas Reynolds numbers. In the end, the most efficient design is the one with the highest mass transfer to power input ratio, where the power input is a function of the pressure drop, gas velocity and shaft power.

30 While stripping, absorption and distillation have been studied in most of the aforementioned HiGee contactors and some related industrial applications have been implemented (see section 6), limited research has been conducted on solid-catalyzed reactions ${ }^{67-71}$ and/or catalytic 
not only affect their fractional recovery of solute but also the attainable conversions.

\section{4. Modeling and simulation}

4 When it comes to modeling and simulation, the main differences between a conventional packed column and a HiGee unit are: (1) A packed column is a straight bed while a HiGee may be seen as a tapered bed, in which the cross-sectional area for flow varies along the radial direction, leading to varying gas and liquid velocities. As a result, both gas-side and liquidside transfer coefficients vary along the radius, unlike in straight beds. (2) In a packed bed mass transfer occurs only along the packing while in HiGee an additional mass transfer zone exists between the rotor and the casing. These two differences are, unfortunately, not adequately appreciated in the literature.

A small number of models describing mass-transfer in HiGee gas-liquid contacting processes are available in the literature. Many of them have focused on modeling of absorption and stripping, while just a few on distillation and solid catalyzed stripping. For absorption and stripping, most of the models available are developed based on first principles whereas distillation models usually involve the use of commercial process simulators (e.g. Aspen Plus) and discretization tricks to adapt the available distillation modules to include the effect of centrifugal force and the different flow geometry (radial flow instead of axial flow).

Most HiGee models are developed following a non-equilibrium (NEQ) modelling approach analogous to the one explained in detail by Taylor and Krishna for distillation ${ }^{73}$ and reactive distillation $^{74}$ in conventional columns. In contrast with equilibrium models, non-equilibrium models do not assume that the streams leaving a separation stage are in thermodynamic equilibrium. Instead, NEQ models consider phase equilibrium only at the gas-liquid interphase and therefore take into account that the mass transfer of components from one phase to the other occurs at a certain rate, which is proportional to the concentration gradient. Since phase equilibrium is only assumed to exist at the interphase, separate balance equations are written for all components and for each phase. The resulting balance equations are often called MERSHQ equations (where $\mathrm{M}=$ material balances, $\mathrm{E}=$ energy balances, $\mathrm{R}=$ massand heat-transfer rate equations, $\mathrm{S}=$ summation equations, $\mathrm{H}=$ hydraulic equations for the pressure drop, and $\mathrm{Q}=$ equilibrium equations). ${ }^{73}$

When modeling HiGee contactors, the MERSHQ equations are used to write balances over a differential element with radial length $(\mathrm{dR})$ and surface area $(2 \pi \mathrm{Rh})$. Three different theories are often used to describe mass transfer, they are: film theory ${ }^{75,76}$, penetration theory ${ }^{77}$ and 
1 component material balances ${ }^{74,79}$. If a solid catalyst is used, the possible effect of intraparticle

2 diffusional limitations is accounted for by introducing effectiveness factors when calculating 3 the actual reaction rate ${ }^{79}$. For heterogeneous reactions, such as solid catalyzed reactions, the overall rate of reaction can be limited by mass transfer of the reactants from their respective phases to the active sites of the catalyst. The overall resistance to mass transfer and resistance can be written as the sum of mass transfer resistances in series ${ }^{80}$. For instance, for a solidcatalyzed first order reaction in which gas phase reactant $\mathrm{A}$ dissolves in a liquid phase and then diffuses through the liquid and solid catalyst, where it reacts, the overall rate of reaction can be written as:

$$
-r_{A}=\left(\frac{R T}{H k_{G} a_{G L}}+\frac{1}{k_{L} a_{G L}}+\frac{1}{k_{S} a_{L S}}+\frac{1}{\eta k_{r} C_{C a t}}\right)^{-1} \frac{R T C_{A, G}}{H}
$$

The first three terms in equation (2) account for the resistances to mass transfer of A from the bulk of the gas phase to the liquid-solid interface, and the last term accounts for the resistance to diffusion through the porous catalyst towards the active sites, where reaction occurs. The decrease in the reaction rate due to internal diffusional limitations in the porous catalyst is incorporated by the effectiveness factor.

Depending on the flow arrangement and on whether or not a steady-state assumption is valid, the model can result in a in a few ordinary differential equations or in a system of partial differential equations that must be solved simultaneously to yield the concentrations as a function of the radius and other important parameters. The development of HiGee models for configurations with counter-current flow is usually simpler than for those with cross-current flow. In the former, the flow is mainly in the radial direction and the variation of gas and liquid compositions in the axial direction can be neglected. In the latter, the liquid flows in the radial direction while the gas flows in the axial direction ${ }^{55}$, therefore concentrations change in both directions and the model equations become more complex.

As Table 3 shows, different assumptions with respect to the liquid flow pattern are made when modeling HiGee contactors. The flow pattern assumed has a direct effect on the calculation of the effective surface area available for mass $\operatorname{transfer}^{81}$ and therefore on the model estimate for the mass transfer coefficients. Earlier mass-transfer models, i.e. the one by Munjal et al. ${ }^{82}$, considered that the area available for mass transfer in an RPB was provided by 30 a thin film flowing over some or all or the packing. Burns and Ramshaw ${ }^{83}$ performed a visual study of the liquid flow in an RPB and reported that for rotational speeds between 300 and $600 \mathrm{rpm}$ liquid flow occurs in the pores in the form of radial rivulets whereas for rotational 
1 speeds above 600-800 rpm droplet flow and film flow predominate, with a higher proportion 2 of droplet flow as the rotational speed increases. Based on these observations, more recent 3 models assume droplet flow or a combination of droplet flow and film flow over the packing.

4 Guo et al. ${ }^{56}$ developed a model to describe three types of mass transfer processes (a gas-side mass transfer controlled process, a liquid-side mass transfer controlled process and gas-side mass transfer controlled process with reaction) in a cross-flow RPB. In their work, Guo et al. presented experimental correlations to estimate the droplet diameter and the film thickness as a function of the centrifugal force and the specific area of the packing. Whether or not these correlations represent the complex droplet-droplet and droplet-packing collisions inside the packings for a given system needs to be proven on a case by case basis.

\section{First-principle mass-transfer models}

One of the earliest models for mass transfer in a rotating packed bed based on first principles was developed by Munjal et al. ${ }^{82}$. They used penetration theory and the complete convectiondiffusion model to obtain correlations for the estimation of gas-liquid $\left(\mathrm{k}_{\mathrm{L}}\right)$ and liquid-solid $\left(\mathrm{k}_{\mathrm{s}}\right)$ mass-transfer coefficients in rotating packed beds. The authors approximated the flow in a high gravity packed bed by liquid-film flow along the flat vertical surface of a rotating blade and liquid-film flow along the horizontal surface of a rotating disk. The expressions developed for gas-liquid and liquid-solid mass transfer coefficients on these idealized surfaces were then extended to the correlations for rotating packed beds.

Chen et al.$^{84}$ presented a rigorous dynamic model for the ozonation of a pollutant (o-Cresol). The model considers the simultaneous ozone and oxygen mass transfer, the chemical reactions of ozone self-decomposition, and pollutant ozonation and the effect of chemical reactions on gas-liquid mass transfer. The resulting system of partial differential equations is solved using the finite difference method based on the Taylor series. The model predicts the dynamic variations of ozone, o-cresol and oxygen concentration profiles in RPB.

Sun et al. ${ }^{85}$ developed a model to describe the simultaneous absorption of two gases with a pseudo-first-order reaction between them at the liquid surface in a rotating packed bed. The model was used to estimate the overall volumetric mass-transfer coefficients and it was validated with the simultaneous absorption of $\mathrm{CO}_{2}$ and $\mathrm{NH}_{3}$ into water. They neglected end effects and pressure drop and assumed the liquid flow in the rotor to be in the form of droplet flow in the void and film flow on the packing surface. As a result, the total gas-liquid interfacial area consisted of the surface area of the packing plus the surface area of all of the droplets. They then developed expressions for the liquid side mass transfer coefficients both 
1 in the droplets and the liquid film in terms of the droplet size and the film thickness, 2 respectively. The model took into account the effect of rotational speed on the mass transfer area of the droplets and the liquid film, but assumed these parameters to remain constant along the radial direction. They then used the correlations for $\mathrm{k}_{\mathrm{L}}$, together with Onda's correlation $^{60}$, equation (1), for the gas-side mass transfer coefficient, to calculate the overall mass transfer coefficient using equation (3). The $K_{G} a$ values estimated by the model were found to agree well with the experimental results at various liquid volumetric flow rates, gas volumetric flow rates, rotational speeds, and $\mathrm{NH}_{3} / \mathrm{CO}_{2}$ molar ratios.

$$
\frac{1}{K_{G} a}=\frac{1}{k_{G} a_{G L}}+\frac{H}{k_{L} a_{G L}}=\frac{1}{k_{G} a_{G L}}+\frac{H}{k_{L_{1}} a_{d}+k_{L_{2}} a_{f}}
$$

Here, the area of mass transfer is composed of the surface area of the droplets $\left(a_{d}\right)$ and the surface area of the liquid film over the packing $\left(a_{f}\right)$.

Yi et al. ${ }^{86}$ modeled the gas-liquid mass transfer with reactions for the absorption of $\mathrm{CO}_{2}$ by a Benfield solution in a rotating packed bed. They assumed liquid flow to be in the form of spherical droplets, neglecting laminar film flow at high rotating speeds. In contrast to previous models ${ }^{56,87}$, theirs takes into account the variation in droplet size within the rotor along the radial direction. They went further to divide the rotor into two zones, the end zone and the bulk packing zone, and used correlations developed based on the droplet size measurements done by $\mathrm{Zhang}^{88}$ to calculate the droplet size in each of the zones as a function of centrifugal acceleration. Most of the calculated mole fractions of the $\mathrm{CO}_{2}$ in the outlet gas agreed well with the experimental data with a deviation within $10 \%$. Furthermore, they presented a $\mathrm{K}_{\mathrm{G}}$ a profile along the radial direction of the packing.

Quian et al. ${ }^{89,90}$ developed a reaction-equilibrium-mass transfer model based on penetration theory to describe the selective $\mathrm{H}_{2} \mathrm{~S}$ absorption process in methyldiethanolamine (MDEA). Their model is based on three main assumptions: (1) Liquid flow in the rotating packed bed is laminar film flow only, (2) surface area of packing regarded as gas-liquid effective interfacial area, (3) the rotor in the rotating packed bed consists of a given number of layers and the film is renewed once every time it passes through one layer of packing. They validated the model using industrial scale experimental data obtained in a refinery in China. Solid-catalyzed reactive stripping for the production of octyl-hexanoate with simultaneous water removal from the reaction zone has been modeled by Gudena et al. ${ }^{79}$ Their work included a mathematical model derived from first principles in order to study the diffusional mass transfer within a porous catalyst in an RPB by using the effectiveness factor as a measure of 
1 diffusional resistance. They analyzed the influence of the centrifugal field on the variation of 2 the catalyst effectiveness factor and the selectivity in an esterification reaction. Table 3 shows an overview of some of the first-principle modeling work done on rotating packed beds for gas-liquid processes.

$R P B$ simulations with the aid of process simulators

Besides the mass-transfer models mentioned so far, there are also those developed with the aid of commercial process simulators. However, the fact that these simulators do not include a built-in module to model and simulate HiGee processes is a hurdle. This is especially true when modeling more complex processes such as distillation or reactive systems for which first-principle models can be both difficult and tedious. For this reason, most of the modeling and simulation studies of rotating packed beds reported in the literature ${ }^{91-96}$ have opted to modify existing units (e.g. RADFRAC module in Aspen Plus) so that the special characteristics of HiGee contactors, such as the effect of the centrifugal field on mass transfer coefficients and pressure drop and the variation of flow area with radial length, can be taken into account. The rate-based functionality of Aspen Plus was used in all these cases, allowing for rigorous simulations based on non-equilibrium models, which use rate equations and experimentally obtained correlations for mass transfer, pressure drop and rates of reaction, in the case of reactive systems. It is worth mentioning that most of the mass transfer studies in HiGee have used rotors with inner and outer radii of about 2 and $8 \mathrm{~cm}$, respectively, and have reported average mass transfer coefficients. The use of these data for the simulation of an industrial size HiGee of $0.5 \mathrm{~m}$ radius involves a huge and risky extrapolation due to the substantial variation of the flow area as the radius increases. Local mass transfer coefficients should be used instead in such cases, when the variation coefficient is very large. To the best of our knowledge, only Reddy et al. ${ }^{62}$ have reported local coefficients for their split-packing design which are not based on a broad range of parameters. The correlations developed by Chen et al. ${ }^{97}$ and Chen ${ }^{98}$ (equations (4) and (5)) can be used to estimate liquid-side and gasside mass transfer coefficients since they have been shown to account for end-effects, packing characteristics and the size of HiGee contactors. Their application to any industrial large size HiGee should be done with caution.

$$
\frac{k_{L} a_{G L} d_{p}}{D_{L} a_{t}}\left(1-0.93 \frac{V_{o}}{V_{t}}-1.13 \frac{V_{i}}{V_{t}}\right)=0.35 S c_{L}^{0.5} \operatorname{Re}_{L}^{0.17} G r_{L}^{0.3} W e_{L}^{0.3}\left(\frac{a_{t}}{a_{p}^{\prime}}\right)^{-0.5}\left(\frac{\sigma_{c}}{\sigma_{w}}\right)^{0.14}
$$

$$
\frac{k_{G} a_{G L}}{D_{G} a_{t}^{2}}\left(1-0.9 \frac{V_{o}}{V_{t}}\right)=0.023 \operatorname{Re}_{G}^{1.13} \operatorname{Re}_{L}^{0.14} G r_{G}^{0.31} W e_{L}^{0.07}\left(\frac{a_{t}}{a_{p}^{\prime}}\right)^{1.4}
$$


1 In order to solve the MERSHQ equations for a rotating packed bed using the non-equilibrium 2 approach in Aspen Plus with a RADFRAC unit, the system is discretized in such a way that it takes into account that the flow, mass and heat transfer occur in the radial direction, and not in the axial direction as the process simulator assumes for a conventional packed column. Different discretization methods have been applied. For instance, Gudena et al. ${ }^{94}$ divided a coaxially oriented horizontal RPB into radial segments, as shown in Figure 11, and used a variable transformation based on the conservation of material flux and volume for differential segments within the RPB to convert the differential annular rings into a series of sequentially attached vertical cylinders. For a detailed explanation of the variable transformation employed by Gudena et al., the reader is remitted to their publication, where the methodology is illustrated with the production of methyl acetate by reactive distillation in a RPB. ${ }^{94}$ The same methodology was also used in their subsequent articles about multiple-objective optimization of an RPB for VOC stripping ${ }^{93}$, modeling and optimization of HiGee stripper-membrane systems for bioethanol recovery and purification ${ }^{92}$ and for methyl lactate hydrolysis ${ }^{95}$. These multi-objective optimization problems were formulated to maximize solute recovery while minimizing total annual cost.

Prada et al. ${ }^{91}$ also performed a computational study using the distillation of the ethanol-water system. The simulation was done in Aspen Plus V7 using the rate-based model of the RADFRAC module, with a subroutine in Fortran 11.0 to replace the correlations used by Aspen Plus to calculate the individual mass transfer coefficients with correlations previously developed in the literature for rotating packed beds ${ }^{37}$. However, it is not clear if they modified the model to account for the fact that transport phenomena do not occur in the same direction in a rotating packed bed than in a conventional packed column. The height equivalent of a theoretical plate in the HiGee was calculated to be $0.0055 \mathrm{~m}$ while for the conventional column it was $0.3246 \mathrm{~m}$ for the same separation. Joel et al. ${ }^{96}$ followed a similar approach to the one taken by Prada et al. but they used it to model and simulate a HiGee reactive absorber for post-combustion $\mathrm{CO}_{2}$ capture in monoethanolamine (MEA) instead of distillation. To do so, they modified the rate-based absorber model in Aspen Plus by replacing the default correlations with new ones suitable for RPBs. These correlations were written in Visual Fortran as subroutines and were dynamically linked with the Aspen Plus rate-based absorber model. The model was able to predict the experimental data obtained by Jassim et al. ${ }^{99}$ with a relative error of less than $8 \%$ for almost all the variables assessed. The validated model was then used for process analysis of the 
1 Joel et al. predicted a 12-fold size reduction when using a rotating packed bed absorber 2 instead of the conventional packed bed absorber.

\section{5. Process design and control}

The design of a counter-current HiGee unit differs from that of a conventional packed column in three main aspects: (1) in a HiGee contactor, flow occurs in the radial direction while in a conventional column it occurs in the axial direction, (2) a HiGee contactor has an additional degree of freedom (the rotational speed) and (3) special considerations must be taken into account for proper design of liquid and gas inlets and outlets in HiGee in view of their higher gas-liquid throughputs per unit area ${ }^{100}$.

Contrary to conventional packed columns, the flow in a HiGee contactor takes place in the radial direction and not in the axial direction. For this reason, separation extent is determined by the radial length of the rotor (the distance between the inner and outer radii) while hydraulic capacity is given by the cross-sectional area at the inner periphery of the rotor (Figure 12). ${ }^{50}$

Certain constraints need to be taken into account in order to produce feasible designs. For compactness, the inner radius should be as small as possible, provided that it results in an acceptable exit gas velocity so that the liquid jets emanating from the distributor do not get carried away ${ }^{100}$. In addition to that, it should provide enough space to accommodate the liquid distributor while allowing gas withdrawal from the eye of the rotor without excessive pressure drop $^{100}$. The axial length, on the other hand, should be such that the unit is operated near but below flooding conditions. Too high of an axial length will result in unwetted packing, and therefore in a rotating packed bed that is bulkier than necessary. ${ }^{100}$ Both outer radius and axial length are constrained by mechanical considerations such as bearing loads, vibration moments and by the strength of the packing material and the support basket used to contain the packing. ${ }^{101}$ Sudhoff et al. ${ }^{102}$ provide a list of suggested constraints for rotational speed, inner and outer radii, axial length, number of rotors, superficial gas velocity and pressure drop that can be used during the design of rotating packed beds for distillation. Most of the HiGee contactors reported in the literature have been designed for specific cases and only one systematic design procedure has been reported by Agarwal et al. ${ }^{100}$. Sudhoff et al. ${ }^{102}$ recently complemented the procedure by including equations to calculate power consumption, required equipment space and investment and operating costs for an RPB for distillation, which is useful for analysis during conceptual process design. A simplified workflow of the complete design methodology is shown in their paper. 
1 In the systematic design procedure for HiGee absorption/distillation systems suggested by 2 Agarwal et al. ${ }^{100}$ the authors explain how to calculate the basic design parameters for a 3 rotating packed bed, which are the inner radius $\left(r_{i}\right)$, the outer radius $\left(r_{0}\right)$ and the axial height 4 (h). The paper also contains guidelines for proper liquid distributor design, packing material selection, rotational speed and pressure drop considerations, casing design and power consumption. The design procedure starts by assuming an actual operating reflux (rectification), boil-up (stripping) or liquid to gas (absorption) ratio between 1.2 to 1.5 times the minimum ratio that can be calculated using conventional equilibrium stage techniques (see, e.g., the classic text by Treybal ${ }^{103}$ ), fixing this way the gas (vapor)-liquid load inside the RPB. The next step is to choose an RPB packing material (metal foam, wire mesh, or other) and a rotor type (single block, split packing) and based on this selection, impose constraints on the maximum permissible RPB height (h), and outer radius $\left(\mathrm{r}_{\mathrm{o}}\right)$. After that, a reasonable operating rotational speed is selected and the inner radius is chosen as the lowest radius that can accommodate the liquid distributor while allowing gas withdrawal from the eye of the RPB without an excessive pressure drop and such that the liquid jet to exit gas kinetic energy ratio at the inner periphery of the rotor is above a suggested value of 3. Agarwal et al. ${ }^{100}$ derived equation (6) to calculate the required inner radius:

$$
r_{i, \text { min }}=\left(\frac{G}{\pi v_{j e t}\left(1-f_{d}\right)}\right)^{\frac{1}{2}}\left(\frac{\rho_{g} p}{\rho_{l}}\right)^{\frac{1}{4}}
$$

After the inner radius has been calculated, the axial length, $h$, of the rotating packed bed is then chosen such that process operation at design conditions is only slightly below flooding for the chosen rotational speed. The superficial gas velocity at an approach to flooding between 0.70 and 0.90 is calculated with Wallis correlation, whose rearranged version is shown in equation (7), and is then used to find the RPB axial length as a function of the volumetric gas flow rate with equation (8).

$$
U_{G, i}=\left(\frac{\beta N_{g}^{a} a_{t}^{b} \mu^{c}\left(\rho_{l}-\rho_{g}\right)^{\frac{1}{4}}}{\rho_{g}^{1 / 4}+\lambda\left(\frac{L}{\alpha G}\right)^{1 / 2} \rho_{l}^{1 / 4}}\right)^{2}
$$

$$
h=\frac{G}{2 \pi r_{i} U_{G, i}}
$$

Where the coefficients and powers $(\beta, \mathrm{a}, \mathrm{b}, \mathrm{c}, \lambda)$ in equation (7) vary from system to system. 
1 Finally, the outer radius, $r_{0}$, is determined by the desired degree of separation. This can be 2 done by performing a material balance over a differential annular shell of the RPB for the primary component and integrating it from the inner radius until the desired degree of separation is achieved. Having now calculated the three most important design parameters, the mechanical robustness of the RPB should be checked. If the maximum outer radius and axial length constraint(s) is (are) violated, the gas and liquid feeds should be split into half and the design is repeated for each half. The liquid distributor is designed for each RPB, the total pressure drop is calculated and the compressor/blower are sized accordingly. The casing liquid sump is sized for a liquid hold up of 1-2 min and the motor(s) power consumption for rotating the $\mathrm{RPB}(\mathrm{s})$ is determined. For a more detailed explanation please refer to the original publication by Agarwal et al. ${ }^{100}$, where they demonstrate their procedure with four case studies: n-butane/isobutane distillation, benzene-cumene distillation, natural gas dehydration using TEG and $\mathrm{CO}_{2}$ absorption in DEA, which they compare with their corresponding conventional column distillation processes, estimating total volume reduction factors of 13 , 10,68 and 7 , respectively ${ }^{100}$. It should be noted that the rotating packed beds designed in these case studies had split packing and so the authors used the corresponding hydraulic and mass-transfer coefficient correlations in their procedure. More recently, Sudhoff et al. ${ }^{102,104}$ presented an integrated design methodology for distillation in RPBs, which is based on the procedure presented by Agarwal et al. The method can be used as a tool for feasibility studies for the application of rotating packed beds for distillation. Their integrated design method enables the selection of an appropriate range of operating and design variables to design a highly flexible RPB that can handle a range of feed compositions. For this flexibility analysis, Sudhoff et al. developed a graphical method that uses flexibility maps previously used in other fields but new for chemical processes. They define the degree of flexibility of an RPB as the measure of the range of fluctuations in the feed composition that can still be compensated by varying the rotational speed of the RPB without changing the product specifications. In their methodology, the design parameters $\left(h, r_{i}, r_{o}\right)$ of an RPB are calculated following a similar approach to the one developed by Agarwal et al. ${ }^{100}$ and previously described. However, the design parameters thus calculated are not taken as the optimal design values but rather as initial values, since they do not necessarily offer the biggest flexibility. The effect of variations (both positive and negative) of these initially calculated design parameters on the flexibility of the RPB is studied independently to find new values for $h, r_{i}$ and $r_{o}$ that lead to a highly flexible design. All of this, off course, taking 
1 into account that the RPB flexibility changes with its dimensions, which have an effect on

2 both investment and operating costs and are finally reflected in the cost per ton of product.

3 Concerning the process control of HiGee, the literature is practically absent on this topic.

4 Because of the high throughput and low inventory, HiGee contactors respond much faster to feed composition variations, and feed flow variations. The control strategy for HiGee distillation should depend on the analysis frequency: if a fast inline measurement of composition is possible (direct by RI/UV-VIS, NMR) then the reboiler and condenser volume should be as low as possible to have a fast response. If a slow "off-line" measurement is done (GC/HPLC), then the condenser and reboiler volume should be higher, to dampen fluctuations and allow for timely control. The first case is obviously desired since the whole idea of the $\mathrm{RPB}$ is to reduce volumes and inventories.

In the same way, scarce information about process economics of HiGee distillation is available in the literature. Lower investment and operating costs are reported for some of the industrial applications discussed in section 6 of this paper. However, those figures depend not only on the dimensions of the rotating bed, reboiler and condenser, but also on undisclosed variables affecting each specific case. Therefore they cannot always be used for cost estimates of other processes. For a calculation basis for investment and operating costs of rotating packed beds based on similarities, the reader is also referred to Sudhoff et al. ${ }^{102}$.

\section{Industrial applications}

To the best of our knowledge, the only reported industrial applications of HiGee for gasliquid contacting processes are: seawater de-aeration, reactive stripping of hypochlorous acid, $\mathrm{SO}_{2}$ removal, selective absorption of $\mathrm{H}_{2} \mathrm{~S}$, water de-aeration for softdrink bottling and distillation. These applications are summarized in Table 4 and described hereafter.

\subsection{Seawater de-aeration}

The first commercial application of HiGee to be reported was the seawater de-aeration at the Shengli Oil Field of China Petrochemical Corporation ${ }^{105}$, where rotating strippers with a 1.4 m diameter replaced a $32 \mathrm{~m}$ high vacuum tower system. In this plant, two HiGee units with a water throughput per unit of $250 \mathrm{t} / \mathrm{h}$ were used to reduce dissolved oxygen in the seawater from 6-12 ppm to less than $50 \mathrm{ppb}$. The HiGee units replaced a high vacuum tower system that had a lower oxygen removal efficiency and therefore required additional chemical treatment of the water to achieve the desired oxygen levels. 


\subsection{Reactive stripping of hypochlorous acid}

In 1999, Dow Chemicals successfully introduced one of the first commercial applications of rotating packed beds, the production of hypochlorous acid $(\mathrm{HOCl})^{32,106}$. In this reaction, chlorine is absorbed into an aqueous solution of sodium hydroxide and reacts instantaneously to produce sodium chloride and $\mathrm{HOCl}$, the desired product. In the presence of sodium chloride, the $\mathrm{HOCl}$ quickly decomposes to sodium chlorate $\left(\mathrm{NaClO}_{3}\right)$, an undesired byproduct, reducing the product yield. Due to the fast kinetics of both $\mathrm{HOCl}$ formation and decomposition reactions, the chlorine absorption-reaction step is liquid-side mass transfer limited while desorption of $\mathrm{HOCl}$ is gas-side mass transfer limited. Taking into account the short residence time and high mass transfer rates offered by rotating packed bed technology, Trent and Tirtowidjojo ${ }^{32}$ designed a process for the production of $\mathrm{HOCl}$ through reactive stripping in a rotating packed bed. In this process, the aqueous solution of sodium hydroxide is introduced at the eye of the rotor and then moves radially outwards while contacting the chloride gas stream countercurrently. The $\mathrm{HOCl}$ produced is quickly stripped into the gas phase and removed from the reaction zone before it decomposes to chlorate. Thanks to the intensified mass transfer and the low residence time, chlorine absorption-reaction and $\mathrm{HOCl}$ desorption both take place quickly, reducing $\mathrm{HOCl}$ decomposition and leading to $\mathrm{HOCl}$ yields of more than $10 \%$ higher than those achieved with the conventional process, which was unable to reach a yield of $80 \%$. Additionally, less than half the amount of stripping gas is necessary while a 40 -fold reduction in equipment size is achieved compared with the conventional spray tower technology. In 2003, Trent and Tirtowidjojo ${ }^{32}$ reported that after two and a half years of operation the rotating packed bed had consistently maintained these and even better yields, while proving to be mechanically reliable. They also stated that start up and shut down was easy and with little maintenance required.

\section{3. $\mathrm{SO}_{2}$ removal}

$\mathrm{SO}_{2}$ is a major pollutant emitted from the combustion of fossil fuels, which is hazardous to human health and contributes to the formation of acid rain. In the ammonia-based wet scrubbing process, which is one of the desulfurization methods most widely applied in China, the absorbent solution containing ammonium sulfite reacts with the $\mathrm{SO}_{2}$ absorbed, removing it from the acid gas ${ }^{107}$. However, due to poor mass transfer efficiency, this process requires large packed columns or spray towers, leading to high capital and operating costs ${ }^{108}$. Rotating packed beds can then be used to intensify mass transfer and reduce the size of the columns. 
1 In 1999, a rotating packed bed absorber with a capacity of $3000 \mathrm{~m}^{3} / \mathrm{h}$ of gas was installed at 2 the Zibo Sulphuric acid plant (Shandong province, China) in parallel to the existing tower 3 system for tail gas cleaning of $\mathrm{SO}_{2}{ }^{109}$. During the absorption tests, $\mathrm{SO}_{2}$ concentrations in the tail gas of less than $300 \mathrm{ppm}$ (or even as low as $50 \mathrm{ppm})^{26}$ were achieved, while capital investment, volume and energy consumption substantially decreased in comparison to the conventional tower system (see Table 4).

\subsection{Selective absorption of $\mathrm{H}_{2} \mathrm{~S}$}

$\mathrm{H}_{2} \mathrm{~S}$ needs to be removed during the gas treating process of refinery gas, syngas or natural gas. However, these gas streams normally contain big amounts of $\mathrm{CO}_{2}$ that, if co-absorbed, affect the performance of desulfurization process by increasing the solvent circulation rate and the energy load of the solvent regeneration system. Moreover, the $\mathrm{CO}_{2}$ present in the desorbed acid gas dilutes the $\mathrm{H}_{2} \mathrm{~S}$ in the feed stream going into the Claus unit, hindering the efficiency of the sulfur recovery system. For these reasons, selective $\mathrm{H}_{2} \mathrm{~S}$ removal is a subject of high interest for the oil and gas industry ${ }^{89}$.

The selective desulfurization process most widely used in refineries is based on the chemical absorption of $\mathrm{H}_{2} \mathrm{~S}$ in methyldiethanolamine (MDEA) ${ }^{89}$. MDEA is thermodynamically selective towards $\mathrm{CO}_{2}$ but kinetically selective towards $\mathrm{H}_{2} \mathrm{~S}$ and, as a result, long gas-liquid contact times in conventional packed columns promote $\mathrm{CO}_{2}$ co-absorption. Therefore, the combination of low residence time and high mass transfer rates offered by rotating packed beds favor the selective absorption of $\mathrm{H}_{2} \mathrm{~S}^{89}$. Fujian Petroleum Refinery Co. (China) installed a rotating packed bed to replace a packed bed column for the selective absorption of $\mathrm{H}_{2} \mathrm{~S}$ over $\mathrm{CO}_{2}$ using MDEA as a solvent. As a result, the $\mathrm{CO}_{2}$ co-absorption was reduced from $79.9 \%$ to $8.9 \%$ while the equipment volume drastically reduced from $36 \mathrm{~m}^{3}$ to $3.4 \mathrm{~m}^{3}$. Details are shown in Table 4.

\subsection{Water de-aeration for soft drink bottling}

In 2006, GasTran installed their first RPB vacuum de-aeration system at PepsiAmericas to remove dissolved oxygen from water and thus reduce foaming and increase bottling line speed and product quality in their carbonated soft drink bottling process ${ }^{110}$. In the GasTran vacuum de-aeration system water is fed through the center of the rotor, in which very small water droplets with a large specific surface area are produced and exposed to the vacuum, allowing gas desorption and removal to occur. The de-aerated water is collected and exits through the bottom of the casing while the dissolved gases are desorbed and exit through the 
1 top of the bed towards a vacuum pump. No stripping gas is required to achieve dissolved 2 oxygen levels in the 200 to $500 \mathrm{ppb}$ DO range. GasTran reported increased filling speeds ranging from $10 \%$ to $40 \%$, improvement in the distribution of carbonation levels and increased fill accuracy resulting in less variation in net contents of the final product and in the reduction of reject rates due to low fills. PepsiAmericas purchased their second GasTran Vacuum Deaeration System in early 2008, confirming thus the success of the first system.

\subsection{HiGee distillation}

Besides the aforementioned applications, Wang et al. ${ }^{27}$ reported the commercialization of about 200 rotating zigzag units up to 2011, for the separation of alcohol/water, acetone/water, DMSO/water, DMF/water, ethyl acetate/Water, methanol/tert-butanol, dichloromethane/silylethers, methanol/formaldehyde/water, methanol/toluene/water, ethyl acetate/toluene/water, methanol/methylal/water, methanol/DMF/water. Table 5 shows a list of commercial suppliers of HiGee technology for distillation, absorption and other processes.

It must be highlighted that no industrial applications involving heterogeneous catalysis, i.e., solid-catalyzed reactions or catalytic distillation in HiGee, have been reported so far. This is not surprising since most experimental work has focused on gas-liquid mass transfer while very few ${ }^{67-71}$ on effect of high gravity on liquid-solid mass transfer.

This list of applications just described is very limited considering the large amount of studies (see Table 1) that have been conducted to demonstrate the functionality of HiGee contactors for stripping, distillation, absorption, homogeneous gas-liquid reactions and even solidcatalyzed gas-liquid reactions. The main reason for the slow deployment of these technologies in the industry still seems to be the concerns about the energy use and reliability of these rotating machines, even though the rotating speeds at which they operate is closer to that of pumps and fans than that of high speed centrifuges ${ }^{111}$, all of which are widely used in the industry. The main drawbacks of HiGee technology include: rotating equipment, additional energy requirements (electricity), problems related to the reliability and longevity due to the moving parts, mechanical stability issues, complicated hydrodynamics, seals and bearings.

\section{Conclusions}

This review paper provided an overview of the state-of-the-art in the field of HiGee contactors used for gas-liquid mass transfer processes. Different HiGee contactors have been discussed with respect to their advantages and disadvantages, rotor configuration, working 
1 principles, modeling and simulation, design procedures and practical applications. The RZB seems to have the best performance for continuous distillation when compared to other rotating beds. This is mainly due to its higher gas-liquid contact time and to the possibility of increasing the number of separation stages by installing multiple rotors within one casing. However, the single-block RPB has a much simpler rotor and provides a high surface area that can be used for catalyzed reactive systems.

The literature available confirms that HiGee contactors have been used for several gas-liquid processes, such as absorption, stripping and distillation. Besides, while only few studies have incorporated solid catalysts into the packing ${ }^{67-71}$, they have reported favorable results in comparison to conventional processes. This shows the potential that HiGee has for solidcatalyzed gas-liquid reactions ${ }^{67-71}$, not only due to the intensified gas-liquid mass transfer rates but also to the potentially good catalyst wettings at high centrifugal forces. Most of these studies ${ }^{67-71}$ have addressed specific applications but little work has been done to gain fundamental knowledge about solid-catalyzed reactions under high centrifugal forces. One of the main issues that has to be addressed during future research is the very low liquid holdup of HiGee contactors, which reduces the residence time in reactive systems.

Another deficiency in the field is the lack of clear scale-up rules. The variation in flow area and centrifugal force in the radial direction of a HiGee make the average mass transfer coefficients (usually reported in literature) not very useful for industrial scale-up. The use of these data is even more complicated when important information about the type of distributor and packing used and the operating conditions at which experiments were conducted is not disclosed in research articles.

Despite the considerable amount of research carried out so far, most of it has not exploited the full potential of HiGee. Operation at high superficial velocities at a laboratory scale requires such small HiGee units that they can be far from representing industrial scale rotating beds. A trade-off then exists between reaching the maximum capabilities of rotating beds and obtaining scalable results. This trade-off could be eliminated by having more involvement from the industry in HiGee research, making it possible to run experiments in production sites, enabling operating conditions not reachable in a research laboratory.

There are also other issues and challenges ahead that need to be solved, such as: mechanical complexity and reliability, uncertainty in design data, use of catalytic reactions, reliability at high/low pressure/temperature, corrosion resistance of packing materials, dynamic balance of large rotating packed beds, limitation in the number of stages, sensitivity towards initial liquid distribution, limitations in scale of operation (i.e. throughput). 
1 On the other hand, based on the amount of experimental work done and on the list of

2 industrial applications reported in the literature, HiGee technology seems to have already its

3 place in China while it has been relegated in Western countries, where it was first developed.

4 Concerns about the reliability of rotating packed beds may be one of the reasons for this lack

5 of interest, even though Dow Chemicals has reported the successful long-term operation of

6 their RPBs for the production of $\mathrm{HOCl}$ and the fast and simple startup of these machines to

7 perform preventive and corrective maintenance when required.

8 The lack of simulation modules in commercial process simulators is another matter that

9 deserves attention. Without these modules, it is difficult to assess the performance of HiGee technology during conceptual design phases and feasibility studies. This may keep companies from realizing potential benefits of HiGee technology for their processes, resulting in new plants being designed with the same conventional units that have already proven to work for decades instead of taking risks with novel technology.

14 The process industry could benefit from HiGee in several ways. For instance, by having inherently safer designs (with reduced inventories) and by gaining flexibility (due to much lower start-up times that make HiGee technology convenient for production campaigns), as well as switching from batch to continuous processes (without significant production loss). Collaboration between the private sector and academia is then required to develop this promising technology and prove its benefits and long-term reliability in order to overcome the obstacles for its deployment in the industry.

\section{Acknowledgement}

This research was carried out within the HighSinc program - a joint development between AkzoNobel and the Department of Chemical Engineering and Chemistry from Eindhoven University of Technology - where $12 \mathrm{PhD}$ students will work on various aspects and applications of HiGee technologies.

\section{Nomenclature}

$31 \quad$ Symbols

Total specific area of mass transfer, $\mathrm{m}_{\mathrm{i}}^{2} \mathrm{~m}_{\mathrm{R}}^{-3}$ 
$1 a_{d}$

$2 a_{f}$

$3 \quad a_{p}^{\prime}$

$4 a_{t}$

5 ATU

$6 C$

$7 \quad C_{\text {Cat }}$

$8 D_{G}$

$9 \quad d_{p}$

$10 f_{d}$

$11 F-$ factor

$12 G$

$13 \quad \mathrm{Gr}_{\mathrm{G}}$

$14 \mathrm{Gr}_{\mathrm{L}}$

$15 h$

$16 H$

17 HETP

$18 k_{G}$

$19 k_{G} a_{G L}$

$20 \quad k_{L}$

$21 k_{L} a_{G L}$

$23 \quad k_{L_{2}}$

$24 \quad k_{S}$

$25 \quad k_{S} a_{L S}$

$26 K_{G} a$

$27 \quad k_{r}$
Specific area of the droplets, $\mathrm{m}_{\mathrm{i}}{ }^{2} \mathrm{~m}_{\mathrm{R}}^{-3}$

Specific (wetted) area of the packing, $\mathrm{m}_{\mathrm{i}}{ }^{2} \mathrm{~m}_{\mathrm{R}}{ }^{-3}$

Surface area per unit volume of the $2 \mathrm{~mm}$ diameter bead, $\mathrm{m}^{2} \mathrm{~m}^{-3}$

Specific area of the packing, $\mathrm{m}^{2} \mathrm{~m}^{-3}$

Area of a transfer unit, $\mathrm{m}^{2}$

Constant in Onda equation

Catalyst concentration, $\mathrm{mol}_{\mathrm{cat}} \mathrm{m}^{-3}$

Gas diffusivity, $\mathrm{m}^{2} \mathrm{~s}^{-1}$

Diameter or characterstic dimension of packing, $\mathrm{m}$

Fraction of cross-sectional area of RPB eye occupied by distributor(s)

Vapor kinetic energy term, defined by $U_{G} \rho_{G}^{1 / 2}, \mathrm{~m} / \mathrm{s}\left(\mathrm{kg} / \mathrm{m}^{3}\right)^{1 / 2}$

Volumetric gas flow rate, $\mathrm{m}^{3} / \mathrm{s}$

Gas Grashof number, defined by $\mathrm{Gr}_{\mathrm{G}}=\frac{\mathrm{d}_{\mathrm{p}}^{3} \rho_{\mathrm{G}}^{2} \mathrm{a}_{\mathrm{c}}}{\mu_{\mathrm{G}}^{2}}$

Liquid Grashof number, defined by $\mathrm{Gr}_{\mathrm{L}}=\frac{\mathrm{d}_{\mathrm{p}}^{3} \rho_{\mathrm{L}}^{2} \mathrm{a}_{\mathrm{c}}}{\mu_{\mathrm{L}}^{2}}$

Axial height of the bed packing, $m$

Henry constant of a gas in a liquid, $\mathrm{Pa} \mathrm{m}^{3} \mathrm{~mol}^{-1}$

Height equivalent to a theoretical plate, $\mathrm{cm}$

Gas-side (gas-liquid) mass-transfer coefficient, $\mathrm{m}_{\mathrm{G}}{ }^{3} \mathrm{~m}_{\mathrm{i}}^{-2} \mathrm{~s}^{-1}$

Volumetric gas-side mass transfer coefficient, $\mathrm{m}_{\mathrm{G}}{ }^{3} \mathrm{~m}_{\mathrm{R}}{ }^{-3} \mathrm{~s}^{-1}$

Liquid-side (gas-liquid) mass-transfer coefficient, $\mathrm{m}_{\mathrm{L}}^{3} \mathrm{~m}_{\mathrm{i}}^{-2} \mathrm{~s}^{-1}$

Volumetric liquid-side mass transfer coefficient, $\mathrm{m}_{\mathrm{L}}{ }^{3} \mathrm{~m}_{\mathrm{R}}{ }^{-3} \mathrm{~s}^{-1}$

Mass-transfer coefficient in the droplet, $\mathrm{m}_{\mathrm{L}}{ }^{3} \mathrm{~m}_{\mathrm{i}}^{-2} \mathrm{~s}^{-1}$

Mass-transfer coefficient in the film, $\mathrm{m}_{\mathrm{L}}^{3} \mathrm{~m}_{\mathrm{i}}^{-2} \mathrm{~s}^{-1}$

Liquid-solid mass-transfer coefficient, $\mathrm{m}_{\mathrm{L}}^{3} \mathrm{~m}_{\mathrm{i}}^{-2} \mathrm{~s}^{-1}$

Liquid-solid mass-transfer coefficient, $\mathrm{m}_{\mathrm{L}}^{3} \mathrm{~m}_{\mathrm{R}}^{-3} \mathrm{~s}^{-1}$

Overall volumetric mass-transfer coefficient, $\mathrm{m}_{\mathrm{G}}{ }^{3} \mathrm{~m}_{\mathrm{R}}{ }^{-3} \mathrm{~s}^{-1}$

Reaction rate constant, $\mathrm{m}^{3} \mathrm{~mol}_{\mathrm{cat}}{ }^{-1} \mathrm{~s}^{-1}$ 
$\begin{array}{ll}1 & L \\ 2 & N_{g}\end{array}$

$3 p$

$4 \mathrm{R}$

$5 \quad R e_{L}$

$6 \quad R e_{G}$

$7 \quad r_{i}$

$8 r_{o}$

$9 \quad S c_{L}$

$10 S c_{G}$

$11 U_{G}$

$12 U_{G, i}$

$13 v_{\text {jet }}$

$14 \quad V_{i}$

$15 V_{o}$

$16 V_{t}$

17 We
Liquid flow rate, $\mathrm{m}^{3} \mathrm{~s}^{-1}$

Ratio of centrifugal to gravitational acceleration, $\frac{\omega^{2} r_{i}}{g}$

Ratio of liquid jet to exit gas kinetic energy, -

Gas constant, $\mathrm{m}^{3} \mathrm{~Pa} \mathrm{~mol}^{-1} \mathrm{~K}^{-1}$

Gas Reynolds number, defined by $\frac{L}{a_{t} \mu_{L}}$

Gas Reynolds number, defined by $\frac{U_{G} \rho_{G}}{a_{t} \mu_{G}}$

Inner radius of the bed packing, $\mathrm{m}$

Outer radius of the bed packing, $\mathrm{m}$

Liquid Schmidt number, defined by $\frac{\mu_{L}}{\rho_{L} D_{L}}$

Gas Schmidt number, defined by $\frac{\mu_{G}}{\rho_{G} D_{G}}$

Gas superficial velocity, $\mathrm{m}_{\mathrm{G}}{ }^{3} \mathrm{~m}_{\mathrm{R}}^{-2} \mathrm{~s}^{-1}$

Gas superficial velocity at RPB inner radius, $\mathrm{m}_{\mathrm{G}}{ }^{3} \mathrm{~m}_{\mathrm{R}}{ }^{-2} \mathrm{~s}^{-1}$

Liquid distributor jet velocity, $\mathrm{m} \mathrm{s}^{-1}$

Volume inside the inner radius of the bed, $\mathrm{m}^{3}$

Volume between the outer radius of the bed and the stationary housing, $\mathrm{m}^{3}$

Total volume of the RPB, $\mathrm{m}^{3}$

Webber number, defined by $\frac{L^{2}}{\rho_{L} a_{t} \sigma}$

18 Abbreviations

19 BP-RPB: Blade-packing rotating packed bed

20 CD: Corrugated disk

21 CM: Cross meshwork

22 CRB: Cross-flow concentric-baffle rotating bed

23 CFCR-RB: Cross-flow concentric ring rotating bed

24 DEA: Diethanolamine

25 HiGee: High-gravity

26 MDEA: Methyldiethanolamine 
1 MEA: Monoethanolamine

2 MERSHQ: Material balances, energy balances, summation equations, hydraulic equations

3 and equilibrium equations

4 NEQ: $\quad$ Non-equilibrium

5 ppm: Parts per million

6 RPB: $\quad$ Rotating packed bed

$7 \quad$ RZB: $\quad$ Rotating zigzag bed

8 SP-RPB: $\quad$ Rotating packed bed with split packing

9 SS: $\quad$ Stainless steel

10 TEG: Triethylene glycol

11 TSCC-RPB: Two-stage counter-current rotating packed bed

12 VOC: Volatile organic compounds

13 WM: Wire mesh

14 WT: Wave thread

15

16 Greek letters

$17 \alpha, \beta, \lambda \quad$ RPB flooding correlation fitting parameters

$18 \varepsilon_{\mathrm{P}} \quad$ Porosity of packing, -

$19 \eta \quad$ Effectiveness factor, -

$20 \rho \quad$ Density, $\mathrm{kg} \mathrm{m}^{-3}$

$21 \mu \quad$ Fluid viscosity, $\mathrm{kg} \mathrm{m}^{-1} \mathrm{~s}^{-1}$

$22 \sigma \quad$ Surface tension, $\mathrm{kg} \mathrm{s}^{-2}$

$23 \sigma_{c} \quad$ Critical surface tension of packing, $\mathrm{kg} \mathrm{s}^{-2}$

$24 \sigma_{w} \quad$ Surface tension of water, $\mathrm{kg} \mathrm{s}^{-2}$

$25 \omega \quad$ RPB rotational speed, $\operatorname{rad~s}^{-1}$

26

27 Subscripts

$28 \quad a, b, c \quad$ RPB flooding correlation fitting parameters

$29 \mathrm{G} \quad$ Gas

30 L Liquid 


\section{References}

2 1. Ramshaw, C. \& Mallinson, R. H. Mass transfer process. U.S. Patent No. 4,283,255 (1981).

42 2. Elsenhans, A. US820772. Apparatus for purifying gas. (1906).

$5 \quad 3 . \quad$ Schmidt, P. G. US1051016. Gas washer. (1913).

6 4. Placek, A. US1936523. Distilling and fractioning apparatus. (1933).

7 5. Placek, A. US2220675. Apparatus for treating liquids. (1940).

8 6. Placek, A. US2349002. Process of evaporating liquids and apparatus for carrying out the same. (1944).

7. Placek, A. US2281616. Process and apparatus for treating liquids with a gaseous medium. (1942).

8. Kapitza, P. L. U.S. Patent No. 2,593,763. Rectification apparatus. (1952).

9. Pilo, C. W. \& Dahlbeck, S. W. US2941872. Apparatus for intimate contacting of two fluid media having different specific weight. (1960).

10. Pilo, C. W. \& Dahlbeck, S. W. US3034730. Means for the distribution of a liquid on rotating surfaces. (1962).

11. Pilo, C. W. US3415501. Centrifugal contact aparatus. (1968).

12. Podeielniak, W. J. US2003308. Centrifugal fractionating method and apparatus. (1935).

13. Podbielniak, W. J. US2044996. Method of securing countercurrent contact of fluids by centrifugal action. (1936).

14. Podbielniak, W. J. US2093645. Method and apparatus of refining hydrocarbon oil. (1937).

15. Podbielniak, W. J. US2109375. Apparatus for effecting counter current contact between fluids. (1938).

16. Podbielniak, W. J. US2172222. Centrifugal fluid-treating apparatus and seal. (1939).

17. Podbielniak, W. J. US2286157. Method of securing counter-current contact between fluids. (1942).

29 18. Podbielniak, W. J. US2670132. Centrifugal countercurrent contact apparatus. (1954).

30 19. Podbielniak, W. J. US2758783. Centrifugal countercurrent exchange device. (1956).

31 20. Podbielniak, W. J. US2878993. Centrifugal countercurrent contacting method and apparatus. (1959).

33 21. Podbielniak, W. J. US231185. Centrifugal countercurrent contact systems. (1966).

34 22. Podbielniak, W. J. US3233880. Continuous centrifugal vapor-liquid contactor. (1966). 
23. Podbielniak, W. J. \& Todd, D. B. US3344981. Centrifugal apparatus with axiallyextended rotor. (1967).

24. Podbielniak, W. J. US3344983. Combination clarifier and contactor. (1967).

25. Rao, D. P., Bhowal, A. \& Goswami, P. S. Process intensification in rotating packed beds (HiGee): An appraisal. Ind. Eng. Chem. Res. 43, 1150-1162 (2004).

26. Zhao, H., Shao, L. \& Chen, J. F. High-gravity process intensification technology and application. Chem. Eng. J. 156, 588-593 (2010).

27. Wang, G. Q., Xu, Z. C. \& Ji, J. B. Progress on HiGee distillation-Introduction to a new device and its industrial applications. Chem. Eng. Res. Des. 89, 1434-1442 (2011).

28. Burns, J. R., Jamil, J. N. \& Ramshaw, C. Process intensification: Operating characteristics of rotating packed beds - determination of liquid hold-up for a highvoidage structured packing. Chem. Eng. Sci. 55, 2401-2415 (2000).

29. Ramshaw, C. 'HiGee' distillation - An example of process intensification. Chemical Engineer (London) 13-14 (1983).

30. Luo, Y. et al. Characteristics of a two-stage counter-current rotating packed bed for continuous distillation. Chem. Eng. Process. Process Intensif. 52, 55-62 (2012).

31. Chu, G. W. et al. Distillation studies in a two-stage counter-current rotating packed bed. Sep. Purif. Technol. 102, 62-66 (2013).

32. Trent, D. Intensifying the process. Chem. Eng. 742, 30-31 (2003).

33. Pan, Z. Q., Zhang, Y. J., Deng, X. H. \& K. Huang, J. Experimental investigation into mass transfer between liquid and gas in multi-staged spraying rotating packed bed. $J$. South China Univ. Technol. 34 34, 67-71 (2006).

34. Jian, Q., Deng, X. \& Deng, S. Experimental investigation of gas flow resistance and gas-liquid mass-transfer performance in super-gravity force rotating packed bed with wave form disk packing. Huaxue Fanying Gongcheng Yu Gongyi/Chemical React. Eng. Technol. 14, 48 (1998).

35. Chen, Z., Xiong, S. \& Wu, J. Helical rotating absorber. Huagong Xuebao/Journal Chem. Ind. Eng. 46, 388-392 (1995).

36. Chu, G. W. et al. Studies of $\mathrm{CO} 2$ absorption and effective interfacial area in a twostage rotating packed bed with nickel foam packing. Chem. Eng. Process. Process Intensif. 90, 34-40 (2015).

37. Rajan, S., Kumar, M., Ansari, M. J., Rao, D. P. \& Kaistha, N. Limiting gas liquid flows and mass transfer in a novel rotating packed bed (HiGee). Ind. Eng. Chem. Res. 50, 986-997 (2011). 
1 38. Mondal, A., Pramanik, A., Bhowal, A. \& Datta, S. Distillation studies in rotating packed bed with split packing. Chem. Eng. Res. Des. 90, 453-457 (2012).

39. Shivhare, M. K., Rao, D. P. \& Kaistha, N. Mass transfer studies on split-packing and single-block packing rotating packed beds. Chem. Eng. Process. Process Intensif. 71, 115-124 (2013).

40. Wang, G. Q., Xu, O. G., Xu, Z. C. \& Ji, J. B. New HiGee-rotating zigzag bed and its mass transfer performance. Ind. Eng. Chem. Res. 47, 8840-8846 (2008).

41. Wang, G. Q., Xu, Z. C., Yu, Y. L. \& Ji, J. B. Performance of a rotating zigzag bed-A new HiGee. Chem. Eng. Process. Process Intensif. 47, 2131-2139 (2008).

42. Lin, C.-C. \& Jian, G.-S. Characteristics of a rotating packed bed equipped with blade packings. Sep. Purif. Technol. 54, 51-60 (2007).

43. Lin, C. C. \& Lin, Y. C. Mass transfer performance of a rotating packed bed equipped with blade packings in removing methanol and 1-butanol from gaseous streams. Chem. Eng. Process. Process Intensif. 53, 76-81 (2012).

44. Luo, Y. et al. Mass transfer studies in a rotating packed bed with novel rotors: Chemisorption of CO2. Ind. Eng. Chem. Res. 51, 9164-9172 (2012).

45. Sung, W. Der \& Chen, Y. S. Characteristics of a rotating packed bed equipped with blade packings and baffles. Sep. Purif. Technol. 93, 52-58 (2012).

46. Lin, C.-C. \& Kuo, Y.-W. Mass transfer performance of rotating packed beds with blade packings in absorption of CO2 into MEA solution. Int. J. Heat Mass Transf. 97, 712718 (2016).

47. Luo, Y. et al. A two-stage blade-packing rotating packed bed for intensification of continuous distillation. Chinese J. Chem. Eng. 24, 109-115 (2016).

48. Li, Y. et al. Distillation in a counterflow concentric-ring rotating bed. Ind. Eng. Chem. Res. 53: 4821-4837 (2014).

49. Wang, G. Q., Guo, C. F., Xu, Z. C., Li, Y. M. \& Ji, J. B. A new cross flow rotating bed, part 1: Distillation performance. Ind. Eng. Chem. Res. 53, 4030-4037 (2014).

50. Kiss, A. A. Distillation technology - Still young and full of breakthrough opportunities. J. Chem. Technol. Biotechnol. 89, 479-498 (2014).

51. TU Dortmund website. Available at: http://www.fvt.bci.tudortmund.de/cms/en/research/research_topics/PI/index.html. (Accessed: 23rd August 2016)

52. Chen, Y., Lin, C. \& Liu, H. Mass transfer in a rotating packed bed with various radii of the bed. Ind. Eng. Chem. Res. 44, 7868-7875 (2005). 
53. Yang, K. et al. Determination of the effective interfacial area in rotating packed bed. Chem. Eng. J. 168, 1377-1382 (2011).

54. Reay, D., Ramshaw, C. \& Harvey, A. in Process intensification: engineering for efficiency, sustainability and flexibility 205-249 (Elsevier, 2013). doi:http://dx.doi.org/10.1016/B978-0-08-098304-2.00006-7

55. Chen, Y. S., Hsu, Y. C., Lin, C. C., Tai, C. Y. Der \& Liu, H. S. Volatile organic compounds absorption in a cross-flow rotating packed bed. Environ. Sci. Technol. 42, 2631-2636 (2008).

56. Guo, F., Zheng, C., Guo, K., Feng, Y. \& Gardner, N. C. Hydrodynamics and mass transfer in cross-flow rotating packed bed. Chem. Eng. Sci. 52, 3853-3859 (1997).

57. Lin, C. C. \& Chen, B. C. Characteristics of cross-flow rotating packed beds. J. Ind. Eng. Chem. 14, 322-327 (2008).

58. Jiao, W. Z., Liu, Y. Z. \& Qi, G. S. Gas pressure drop and mass transfer characteristics in a cross-flow rotating packed bed with porous plate packing. Ind. Eng. Chem. Res. 49, 3732-3740 (2010).

59. Sandilya, P., Rao, D. P., Sharma, A. \& Biswas, G. Gas-phase mass transfer in a centrifugal contactor. Ind. Eng. Chem. Res. 40, 384-392 (2001).

60. Onda, K., Takeuchi, H. \& Okumoto, Y. Mass transfer coefficients between gas and liquid phases in packed columns. J. Chem. Eng. Japan 1, 56-62 (1968).

61. Chandra, A., Goswami, P. S. \& Rao, D. P. Characteristics of flow in a rotating packed bed (HiGee) with split packing. Ind. Eng. Chem. Res. 44, 4051-4060 (2005).

62. Reddy, K. J., Gupta, A., Rao, D. P. \& Rama, O. P. Process intensification in a HiGee with split packing. Ind. Eng. Chem. Res. 45, 4270-4277 (2006).

63. Ji, J., Xu, Z. \& Yu, Y. U.S. Patent No. 7,344,126 B2. Equipment of multi-rotors zigzag high-gravity rotating beds. (2008).

64. Li, Y. et al. Rotating zigzag bed as trayed HiGee and its power consumption. Asia-Pac. J. Chem. Eng. 8, 494-506 (2013).

65. Lin, C. C. \& Chu, C. R. Feasibility of carbon dioxide absorption by $\mathrm{NaOH}$ solution in a rotating packed bed with blade packings. Int. J. Greenh. Gas Control 42, 117-123 (2015).

66. Nascimento, J. V. S., Ravagnani, T. M. K. \& Pereira, J. A. F. R. Experimental study of a rotating packed bed distillation column. Brazilian J. Chem. Eng. 26, 219-226 (2009).

67. Chen, Y. H. et al. Biodiesel production in a rotating packed bed using $\mathrm{K} / \gamma-\mathrm{Al} 2 \mathrm{O} 3$ solid catalyst. J. Taiwan Inst. Chem. Eng. 42, 937-944 (2011). 
68. Dhiman, S. K., Verma, V., Rao, D. P. \& Rao, M. S. Process intensification in a tricklebed reactor: Experimental studies. AIChE J. 51, 3186-3192 (2005).

69. Chang, C. C. et al. Combined photolysis and catalytic ozonation of dimethyl phthalate in a high-gravity rotating packed bed. J. Hazard. Mater. 161, 287-293 (2009).

70. Chang, C. C. et al. Pt-catalyzed ozonation of aqueous phenol solution using highgravity rotating packed bed. J. Hazard. Mater. 168, 649-655 (2009).

71. Chen, J., Liu, Y. \& Zhang, Y. Control of product distribution of Fischer-Tropsch synthesis with a novel rotating packed-bed reactor: From Diesel to light olefins. Ind. Eng. Chem. Res. 51, 8700-8703 (2012).

72. Shi, Q., Zhang, P., Chu, G. \& Chen, J. A new high gravity catalytic reactive distillation process for n-butyl acetate synthesis (in Chinese). Beijing Huagong Daxue Xuebao (Ziran Kexueban)/Journal Beijing Univ. Chem. Technol. (Natural Sci. Ed. 38, 5-9 (2011).

73. Taylor, R., Krishna, R. \& Kooijman, H. Real-world modeling of distillation. Chem. Eng. Prog. 99, 28-39 (2003).

74. Taylor, R. \& Krishna, R. Modelling reactive distillation. Chem. Eng. Sci. 55, 51835229 (2000).

75. Nernst., W. Theorie der Reaktionsgeschwindigkeit in heterogenen Systemen. Z. phys. Chem. 47, 52-55 (1904).

76. Whitman, W. G. A preliminary experimental confirmation of the two-film theory of gas absorption. Chem. Metall. Eng. 29, 146-148 (1923).

77. Higbie, R. The rate of absorption of a pure gas into still liquid during short periods of exposure. Trans. Am. Inst. Chem. Eng 35, 36-60 (1935).

78. Danckwerts, P. V. Significance of liquid-film coefficients in gas absorption. Ind. Eng. Chem. 43, 1460-1467 (1951).

79. Gudena, K., Rangaiah, G. P. \& Lakshminarayanan, S. Modeling and analysis of solid catalyzed reactive HiGee stripping. Chem. Eng. Sci. 80, 242-252 (2012).

80. H.S. Fogler. in Elements of Chemical Reaction Engineering. Ch. 12, Diffusion and reaction 813-852 (Prentice Hall Int., 2006).

81. Luo, Y. et al. Investigation of effective interfacial area in a rotating packed bed with structured stainless steel wire mesh packing. Chem. Eng. Sci. (2016). doi:10.1016/j.ces.2016.10.023

82. Munjal, S., Dudukovic, M. P. \& Ramachandran, P. Mass-transfer in rotating packed beds-I. Development of gas-liquid and liquid-solid mass-transfer correlations. Chem. 
Eng. Sci. 44, 2245-2256 (1989).

83. Burns, J. R. \& Ramshaw, C. Process intensification: Visual study of liquid maldistribution in rotating packed beds. Chem. Eng. Sci. 51, 1347-1352 (1996).

84. Chen, Y. H. et al. Modeling ozonation process with pollutant in a rotating packed bed. Ind. Eng. Chem. Res. 44, 21-29 (2005).

85. Sun, B.-C. et al. Simultaneous absorption of $\mathrm{CO} 2$ and NH3 into water in a rotating packed bed. Ind. Eng. Chem. Res. 48, 11175-11180 (2009).

86. Yi, F., Zou, H. K., Chu, G. W., Shao, L. \& Chen, J. F. Modeling and experimental studies on absorption of $\mathrm{CO} 2$ by Benfield solution in rotating packed bed. Chem. Eng. J. 145, 377-384 (2009).

87. Kang, J. L., Sun, K., Wong, D. S. H., Jang, S. S. \& Tan, C. S. Modeling studies on absorption of $\mathrm{CO} 2$ by monoethanolamine in rotating packed bed. Int. J. Greenh. Gas Control 25, 141-150 (2014).

88. Zhang, J. An Experimental and Simulation Study on Liquid Flowing and Mass Transfer in RPB, Ph.D. Dissertation. (Beijing University of Chemical Technology, Beijing, China, 1996).

89. Qian, Z., Li, Z.-H. \& Guo, K. Industrial applied and modeling research on selective H2S removal using a rotating packed bed. Ind. Eng. Chem. Res. 51, 8108-8116 (2012).

90. Qian, Z., Xu, L., Li, Z., Li, H. \& Guo, K. Selective absorption of H2S from a gas mixture with $\mathrm{CO} 2$ by aqueous $\mathrm{N}$-methyldiethanolamine in a rotating packed bed. Ind. Eng. Chem. Res. 49, 6196-6203 (2010).

91. Prada, R. J., Martínez, E. L. \& Maciel, M. R. W. Computational study of a rotating packed bed distillation column. Comput. Aided Chem. Eng. 30, 1113-1117 (2012).

92. Gudena, K., Rangaiah, G. P. \& Lakshminarayanan, S. HiGee stripper-membrane system for decentralized bioethanol recovery and purification. Ind. Eng. Chem. Res. 52, 4572-4585 (2013).

93. Gudena, K., Rangaiah, G. P. \& Lakshminarayanan, S. Optimal design of a rotating packed bed for VOC stripping from contaminated groundwater. Ind. Eng. Chem. Res. 51, 835-847 (2012).

94. Krishna, G., Min, T. H. \& Rangaiah, G. P. Modeling and analysis of novel reactive HiGee distillation. 11th International Symposium on Process Systems Engineering 31, (Elsevier B.V., 2012).

95. Gudena, K., Rangaiah, G. P. \& Samavedham, L. Modeling and optimization of reactive HiGee stripper-membrane process for methyl lactate hydrolysis. Ind. Eng. Chem. Res. 
52, 7795-7802 (2013).

96. Joel, A. S., Wang, M., Ramshaw, C. \& Oko, E. Process analysis of intensified absorber for post-combustion $\mathrm{CO} 2$ capture through modelling and simulation. Int. J. Greenh. Gas Control 21, 91-100 (2014).

97. Chen, Y. et al. Packing characteristics for mass transfer in a rotating packed bed. 45, 6846-6853 (2006).

98. Chen, Y. S. Correlations of mass transfer coefficients in a rotating packed bed. Ind. Eng. Chem. Res. 50, 1778-1785 (2011).

99. Jassim, M. S., Rochelle, G., Eimer, D. \& Ramshaw, C. Carbon dioxide absorption and desorption in aqueous monoethanolamine solutions in a rotating packed bed. Ind. Eng. Chem. Res. 46, 2823-2833 (2007).

100. Agarwal, L., Pavani, V., Rao, D. P. \& Kaistha, N. Process intensification in HiGee absorption and distillation: Design procedure and applications. Ind. Eng. Chem. Res. 49, 10046-10058 (2010).

101. Singh, S. P. et al. Removal of volatile organic compounds from groundwater using a rotary air stripper. Ind. Eng. Chem. Res. 31, 574-580 (1992).

102. Sudhoff, D., Leimbrink, M., Schleinitz, M., Górak, A. \& Lutze, P. Modelling, design and flexibility analysis of rotating packed beds for distillation. Chem. Eng. Res. Des. 94, 72-89 (2015).

103. Treybal Robert, E. Mass-transfer operations. (McGraw-Hill Book Company, 1981).

104. Sudhoff, D., Neumann, K. \& Lutze, P. An Integrated Design Method for Rotating Packed Beds for Distillation. 24 European Symposium on Computer Aided Process Engineering 33, (Elsevier, 2014).

105. Chen, J.-F. The recent developments in the HiGee technology. Presented at the GPEEPIC Conference, Venice, Italy. (2009).

106. Quarderer, G. J. et al. Method for synthesis of hypohalous acid. United States Patent. US6048513. (2000).

107. Gao, X. et al. Gas-liquid absorption reaction between (NH4)2SO3 solution and $\mathrm{SO} 2$ for ammonia-based wet flue gas desulfurization. Appl. Energy 87, 2647-2651 (2010).

108. Jiang, X., Liu, Y. \& Gu, M. Absorption of sulphur dioxide with sodium citrate buffer solution in a rotating packed bed. Chinese J. Chem. Eng. 19, 687-692 (2011).

109. Reay, D., Ramshaw, C. \& Harvey, A. in Process intensification: engineering for efficiency, sustainability and flexibility 323-348 (2013). doi:http://dx.doi.org/10.1016/B978-0-08-098304-2.00009-2 
110. Harbold, G. \& Park, J. Using the GasTran deaeration system to achieve low dissolved oxygen levels for superior line speed and product quality: A case study in carbonated soft drink bottling. in Presented at the 7th International Conference on Process Intensification, Albany, NY (2008).

111. Ramshaw, C. The opportunities for exploiting centrifugal fields. Heat Recover. Syst. CHP 13, 493-513 (1993).

112. Dietrich, C., Treichler, D. \& Armstrong, J. An evaluation of rotary air stripping for removal of volatile organics from groundwater. Air Force Engineering \& Services Report (1987). doi:10.1017/CBO9781107415324.004

113. Liu, H.-S., Lin, C.-C., Wu, S.-C. \& Hsu, H.-W. Characteristics of a rotating packed bed. Ind. Eng. Chem. Res. 35, 3590-3596 (1996).

114. Singh, S. P. Air stripping of volatile organic compounds from groundwater: An evaluation of a centrifugal vapor-liquid contactor. (University of Tennessee Knoxville, 1989).

115. Peel, J., Howarth, C. R. \& Ramshaw, C. Process intensification: HiGee seawater deaeration. Chem. Eng. Res. Des. 76, 585-593 (1998).

116. Chen, Y.-S., Lin, C.-C. \& Liu, H.-S. Mass transfer in a rotating packed bed with viscous Newtonian and non-Newtonian fluids. Ind. Eng. Chem. Res. 44, 1043-1051 (2005).

117. Chia-Chang, L. \& Wen-Tzong, L. Removal of an undesired component from a valuable product using a rotating packed bed. J. Ind. Eng. Chem., 12, 455-459 (2006).

118. Li, W. et al. Process intensification of VOC removal from high viscous Media by rotating packed bed. Chinese J. Chem. Eng. 17, 389-393 (2009).

119. Li, W. et al. A mass transfer model for devolatilization of highly viscous media in rotating packed bed. Chinese J. Chem. Eng. 18, 194-201 (2010).

120. Kelleher, T. \& Fair, J. R. Distillation studies in a high-gravity contactor. Ind. Eng. Chem. Res. 35, 4646-4655 (1996).

121. Lin, C.-C., Ho, T.-J. \& Liu, W.-T. Distillation in a Rotating Packed Bed. J. Chem. Eng. Japan 35, 1298-1304 (2002).

122. Li, X., Liu, Y., Li, Z. \& Wang, X. Continuous distillation experiment with rotating packed bed. Chinese J. Chem. Eng. 16, 656-662 (2008).

123. Munjal, S., Dudukovic, M. P. \& Ramachandran, P. Mass-transfer in rotating packed beds-II. Experimental results and comparison with theory and gravity flow. Chem. Eng. Sci. 44, 2257-2268 (1989). 
124. Kumar, M. P. \& Rao, D. P. Studies on a high-gravity gas-liquid contactor. Ind. Eng. Chem. Res. 29, 917-920 (1990).

125. Chen, Y.-S. \& Liu, H.-S. Absorption of VOCs in a rotating packed bed. Ind. Eng. Chem. Res. 41, 1583-1588 (2002).

126. Lin, C.-C., Liu, W.-T. \& Tan, C.-S. Removal of carbon dioxide by absorption in a rotating packed bed. Ind. Eng. Chem. Res. 42, 2381-2386 (2003).

127. Cheng, H. H. \& Tan, C. S. Reduction of $\mathrm{CO} 2$ concentration in a zinc/air battery by absorption in a rotating packed bed. J. Power Sources 162, 1431-1436 (2006).

128. Chiang, C. Y. et al. Absorption of ethanol into water and glycerol/water solution in a rotating packed bed. J. Taiwan Inst. Chem. Eng. 40, 418-423 (2009).

129. Li, Y., Liu, Y., Zhang, L., Su, Q. \& Jin, G. Absorption of NOx into nitric acid solution in rotating packed bed. Chinese J. Chem. Eng. 18, 244-248 (2010).

130. Lin, C. C. \& Liu, W. T. Ozone oxidation in a rotating packed bed. J. Chem. Technol. Biotechnol. 78, 138-141 (2003).

131. Chen, Y. H. et al. Modeling ozone contacting process in a rotating packed bed. Ind. Eng. Chem. Res. 43, 228-236 (2004).

132. Chiu, C. Y., Chen, Y. H. \& Huang, Y. H. Removal of naphthalene in Brij 30containing solution by ozonation using rotating packed bed. J. Hazard. Mater. 147, 732-737 (2007).

133. Chen, Y.-H., Huang, Y.-H., Lin, R.-H. \& Shang, N.-C. A continuous-flow biodiesel production process using a rotating packed bed. Bioresour. Technol. 101, 668-673 (2010).

134. Boodhoo, K. V. K., Cartwright, C. D., Vicevic, M., Prieto, M. A. \& Tortajada, M. Development of a HiGee bioreactor (HBR) for production of polyhydroxyalkanoate: Hydrodynamics, gas-liquid mass transfer and fermentation studies. Chem. Eng. Process. Process Intensif. 49, 748-758 (2010).

135. Lin, C. C., Wei, T. Y., Hsu, S. K. \& Liu, W. T. Performance of a pilot-scale cross-flow rotating packed bed in removing VOCs from waste gas streams. Sep. Purif. Technol. 52, 274-279 (2006).

136. Lin, C. C. \& Chen, Y. W. Performance of a cross-flow rotating packed bed in removing carbon dioxide from gaseous streams by chemical absorption. Int. J. Greenh. Gas Control 5, 668-675 (2011).

137. Liu, Y., Gu, D., Xu, C., Qi, G. \& Jiao, W. Mass transfer characteristics in a rotating packed bed with split packing. Chinese J. Chem. Eng. 23, 868-872 (2015). 
138. Li, Y., Ji, J., Yu, Y., Xu, Z. \& Li, X. Hydrodynamic behavior in a rotating zigzag bed. Chinese J. Chem. Eng. 18, 34-38 (2010).

139. Li, Y. et al. Pressure drop model on rotating zigzag bed as a new high-gravity technology. Ind. Eng. Chem. Res. 52, 4638-4649 (2013).

140. Lin, C. C., Lin, Y. C., Chen, S. C. \& Hsu, L. J. Evaluation of a rotating packed bed equipped with blade packings for methanol and 1-butanol removal. J. Ind. Eng. Chem. 16, 1033-1039 (2010).

141. Lin, C. C. \& Chu, C. R. Mass transfer performance of rotating packed beds with blade packings in carbon dioxide absorption into sodium hydroxide solution. Int. J. Greenh. Gas Control 42, 117-123 (2015).

142. Tsai, C. Y. \& Chen, Y. S. Effective interfacial area and liquid-side mass transfer coefficients in a rotating bed equipped with baffles. Sep. Purif. Technol. 144, 139-145 (2015).

143. Short, H. New mass-transfer find is a matter of gravity. Chem. Eng. 90, 23 (1983).

144. Zhang, Z., Guo, K., Luo, H., Song, J. \& Qian, Z. Characteristics of mass transfer between gas-liquid phases in a HiGee reactor. Chem. Ind. Chem. Eng. Q. 20, 523-530 (2014).

145. Trent, D., Tirtowidjojo, D. \& Quarderer, G. Reactive stripping in a rotating packed bed for the production of hypochlorous acid. in Green A. (Ed) $3^{\text {rd }}$ International Conference on Process Intensification for the Chemical Industry. London: BHR Group, 217-231 (1999).

146. Reay, D., Ramshaw, C. \& Harvey, A. in Process intensification: engineering for efficiency, sustainability and flexibility (ed. Elsevier) 259-321 (2013). doi:http://dx.doi.org/10.1016/B978-0-08-098304-2.00008-0

147. Krishna, G. Modeling and optimization of novel HiGee processes for water and bioproduct applications. PhD. Thesis (National University of Singapore, 2012).

148. GasTrans Systems Website. http://www.gastransfer.com/ Available at: http://www.gastransfer.com/documents/CaseStudy1.pdf. (Accessed: 28th July 2016) 


\section{$1 \quad$ Tables}

2 Table 1. Comparison of HiGee gas-liquid contactors found in the literature

\section{Equipment}

\begin{tabular}{ll}
\hline Single block & Simple single-block rotor design with a \\
Rotating Packed & higher mechanical strength than other \\
Bed (Counter- & rotors. \\
current flow) & Higher mass transfer performance than \\
& conventional packed bed columns. \\
& Single-block rotor offers a high specific \\
& surface area for gas-liquid contact.
\end{tabular}

Single block

Simple single-block rotor.

Rotating Packed

Capable of handling higher gas flow

Bed (Cross-current flow)

Multi-stage spraying rotating packed bed

Rotating packedbed with waveform disk packing Helical rotating bed

Rotating packed bed with split packing
Lower gas flow resistance than $\mathrm{RPB}^{40}$

Longer residence times for both gas and liquid than in an $\mathrm{RPB}^{40}$

Higher tangential slip velocities which may result in higher gas-side mass transfer coefficients ${ }^{61,100}$. Flooding limits comparable to other Higee devices while $a_{e}$ and $k_{\llcorner} a_{e}$ are higher $^{37}$.
Rotating zigzag bed
Disadvantages

Little or no increase in the gas-
side mass-transfer coefficient with respect to fixed bed column ${ }^{59}$. Coaxial multirotor structurally complicated $^{40}$.

Middle-feed streams difficult to be inserted.

Multiple RPBs needed for continuous distillation.

Very short liquid residence time ${ }^{40}$. Requires liquid distributors. ${ }^{63,83}$ Same disadvantages of countercurrent flow RPB

Difficult to coaxially install multiple rotors in one casing ${ }^{40}$

Potential liquid entrained by gas flow despite lower gas frictional resistance ${ }^{40}$

Difficult to coaxially install multiple rotors in one casing ${ }^{40}$

Mass transfer performance lower than that of an RPB due to the small interfacial area ${ }^{40}$

Difficult to coaxially install multiple rotors in one casing ${ }^{40}$

More complex design than single block RPB with two rotors driven by two independent motors ${ }^{39}$. Maximum rotor size limited by mechanical strength of packing ${ }^{39}$. Middle-feed streams difficult to be inserted $^{62,61 .}$

Coaxial multirotor structurally complicated.

Requires liquid distributor ${ }^{61}$ Higher pressure drop than RPB. Higher pressure drop compared to the conventional RPB and power consumption than RPB ${ }^{40,41,64}$. Lower surface area for the gas-
Application

Stripping

Distillation ${ }^{1,120,66,121,122}$

Absorption 1,39,53,59,85,86,99,108,123129

Homogenous gas-liquid reactions ${ }^{84,130-134}$

Solid catalyzed gas-liquid reactions $^{67-71}$

Absorption $55,57,135,136$

N/A

$\mathrm{N} / \mathrm{A}$

Absorption $^{40}$

Stripping ${ }^{39,61,62}$

Distillation ${ }^{38}$

Absorption 37,39,62,137

Distillation $^{40,41}$

Absorption $^{40}$

Stripping ${ }^{138,139}$

Reaction ${ }^{40}$ 
Two-stage

counter-current

rotating packed

bed

Blade packing

rotating packed

bed

Rotating bed with packing and

blades

Rotating bed with blade packing and baffles

Counter-flow concentric-ring rotating bed

Cross-flow concentric-baffle

Higher slip velocity than $\mathrm{RPB}^{45}$. Lower pressure drop than in an RPB and a rotating bed with blade packings ${ }^{45}$.

Higher volumetric gas-side mass transfer coefficients than in a rotating bed with blade packings ${ }^{45}$

Higher gas-liquid throughputs than RZB (5.6 times) ${ }^{48}$.

Lower pressure drop compared with RZB due to the elimination of the stationary baffles ${ }^{48}$.

Higher gas-side mass transfer coefficient compared to RPB ${ }^{48}$. Middle-feed streams can be inserted.

Multirotors can be coaxially installed in one casing.

Lower pressure drop and lower shaft power than RZB ${ }^{49}$.

rotating bed (CRB) Little backmixing.

liquid contacting than $\mathrm{RPB}^{41,40,30}$.

Complex rotor structure with a combination of rotational packing and static rings ${ }^{36}$.

Lower number of theoretical plates per meter under some operation conditions compared with two combined RPBs ${ }^{30}$

Low specific surface area ${ }^{65}$.

Not suitable for solid-catalyzed reactions.

Structure of rotor is more complex than conventional RPB rotor ${ }^{44}$. Lower specific surface area than $\mathrm{RPB}$ and other rotating beds without blades.

Middle-feed streams difficult to be inserted.

Lower specific surface area than RPB and other rotating beds without blades.

Middle-feed streams difficult to be inserted.

Lower mass-transfer performance compared with RZB ${ }^{48}$. Much lower gas-liquid effective interfacial area compared to $\mathrm{RPB}^{48}$.

Stage efficiency of the CRB onethird as much as that of the RZB, or even lower ${ }^{49}$.

\author{
Distillation ${ }^{30,31}$ \\ Absorption $^{36}$ \\ Reactive distillation ${ }^{72}$
}

Absorption $^{45,142}$
Stripping $^{142}$

Distillation $^{48}$ 
1 Table 2. Distillation studies in HiGee contactors

\begin{tabular}{|c|c|c|c|c|c|c|}
\hline System/Type of bed & $\begin{array}{l}\text { Dimensions } \\
\left(r_{i}, r_{o}, h\right)(m)\end{array}$ & $\begin{array}{l}\text { Liquid } \\
\text { flow, } \\
\text { gas flow }\end{array}$ & $\begin{array}{l}\text { Packing } \\
\text { type, a } \\
\left(\mathrm{m}^{2} / \mathrm{m}^{3}\right), \varepsilon_{\mathrm{p}}\end{array}$ & $\begin{array}{l}\text { Rotor } \\
\text { speed } \\
\text { (rpm) }\end{array}$ & $\begin{array}{l}\text { Mass transfer performance } \\
(\mathrm{HETP}, \mathrm{cm}),\left(\mathrm{ATU}, \mathrm{m}^{2}\right), \\
\text { mass transfer coefs. }\end{array}$ & Reference \\
\hline $\begin{array}{l}\text { Methanol/Ethanol } \\
\text { (RPB/ Tot. reflux) }\end{array}$ & $(0.06,0.09,-)$ & $\begin{array}{l}8.42 \times 10^{-3} \\
-8.6 \times 10^{-3} \\
\mathrm{~mol} / \mathrm{m}^{2} \mathrm{~s}\end{array}$ & $\begin{array}{l}\text { SS gauze, } \\
1650,-\end{array}$ & 1600 & $\begin{array}{l}K_{G}=5.4 \times 10^{-5}-44 \times 10^{-5} \\
\mathrm{~mol} / \mathrm{m}^{2} \mathrm{~s} \\
K_{G} a=0.034-0.72 \mathrm{~mol} / \mathrm{m}^{3} \mathrm{~s}\end{array}$ & $\begin{array}{l}\text { (Ramshaw } \\
\text { and } \\
\text { Mallinson, } \\
1981)^{1}\end{array}$ \\
\hline $\begin{array}{l}\text { Ethanol/isopropanol } \\
\text { (RPB) }\end{array}$ & $(0.4,-,-)$ &,-- &,,--- & $\begin{array}{l}1500- \\
3000\end{array}$ & $\mathrm{HETP}=1.75-2.0$ & $\begin{array}{l}\text { (Short, } \\
1983)^{143}\end{array}$ \\
\hline \multirow[t]{2}{*}{$\begin{array}{l}\text { Cyclohexane/n-heptane } \\
\text { (RPB/ Tot. reflux) }\end{array}$} & $\begin{array}{l}(0.0875,0.30 \\
0.15)\end{array}$ & $3.75 \mathrm{~L} / \mathrm{s}$ & $\begin{array}{l}\text { Metal } \\
\text { sponge-like, } \\
2500,0.92\end{array}$ & $\begin{array}{l}400- \\
1200\end{array}$ & $\begin{array}{l}\mathrm{HETP}=3.50-7.50 \\
\mathrm{ATU}=0.04-0.13\end{array}$ & $\begin{array}{l}\text { (Kelleher } \\
\text { and Fair, } \\
1996)^{120}\end{array}$ \\
\hline & & & $\begin{array}{l}\text { Rectangular } \\
\text { packing, } \\
524,0.533\end{array}$ & & & \\
\hline $\begin{array}{l}\text { Methanol/Ethanol } \\
\text { (RPB/Tot. reflux) }\end{array}$ & $\begin{array}{l}(0.0305 \\
0.074 \\
(0.05,0.095))\end{array}$ & - & $\begin{array}{l}\text { SS WM, } \\
982,0.971\end{array}$ & $\begin{array}{l}600- \\
1600\end{array}$ & $\mathrm{HETP}=3.0-9.0$ & $\begin{array}{l}(\text { Lin et al. } \\
2002)^{121}\end{array}$ \\
\hline $\begin{array}{l}\text { Ethanol/water } \\
\text { (RZB/cont. distill.) }\end{array}$ & $\begin{array}{l}(0.2,0.63 \\
0.08)\end{array}$ & - & $\begin{array}{l}\text { Concentric } \\
\text { baffles,-,- }\end{array}$ & $\begin{array}{l}600- \\
1400\end{array}$ & $\mathrm{HETP}=2.9-5.4$ & $\begin{array}{l}\text { (Wang et al., } \\
\text { 2008b) }\end{array}$ \\
\hline $\begin{array}{l}\text { Methanol/water } \\
\text { (RZB/cont. distill.) }\end{array}$ & $\begin{array}{l}(0.1,0.52 \\
0.078)\end{array}$ & - & $\begin{array}{l}\text { Concentric } \\
\text { baffles,-,- }\end{array}$ & $\begin{array}{l}800- \\
2000\end{array}$ & $\mathrm{HETP}=4.0-5.0$ & $\begin{array}{l}\text { (Wang et al., } \\
2008 \mathrm{a})^{40}\end{array}$ \\
\hline \multirow[t]{3}{*}{$\begin{array}{l}\text { Ethanol/water } \\
\text { (RPB/cont. distill.) }\end{array}$} & $\begin{array}{l}(0.03,0.055 \\
0.063)\end{array}$ & $\begin{array}{l}0-30 \\
L / h,-\end{array}$ & $\begin{array}{l}\text { SS CD, 400, } \\
0.82\end{array}$ & $0-1830$ & $\mathrm{HETP}=1.34-2.54$ & $\begin{array}{l}(\text { Li et al.., } \\
2008)^{122}\end{array}$ \\
\hline & & & $\begin{array}{l}\text { SS CM, } \\
1750,0.86\end{array}$ & & $\mathrm{HETP}=1.46-2.50$ & \\
\hline & & & $\begin{array}{l}\text { SS WT, } \\
350,0.95\end{array}$ & & $\mathrm{HETP}=1.02-2.36$ & \\
\hline \multirow[t]{3}{*}{$\begin{array}{l}\text { n-hexane/n-heptane } \\
\text { (RPB/ Tot. reflux) }\end{array}$} & $\begin{array}{l}(0.022,0.08 \\
0.04)\end{array}$ & $\begin{array}{l}5.7-29.0 \\
\mathrm{~cm}^{3} / \mathrm{s},-\end{array}$ & $\begin{array}{l}\text { Raschig } \\
\text { rings, } 627 \text {, } \\
0.62\end{array}$ & $\begin{array}{l}300- \\
2500\end{array}$ & ATU $=0.013-0.027$ & $\begin{array}{l}\text { (Nascimento } \\
\text { et al., } \\
2009)^{66}\end{array}$ \\
\hline & & $\begin{array}{l}5.7-29.0 \\
\mathrm{~cm}^{3} / \mathrm{s},-\end{array}$ & $\begin{array}{l}\text { Raschig } \\
\text { rings, } 765, \\
0.55\end{array}$ & & ATU $=0.012-0.026$ & \\
\hline & & $\begin{array}{l}2.0-18.5 \\
\mathrm{~cm}^{3} / \mathrm{s},-\end{array}$ & $\begin{array}{l}\text { SS WM, } \\
2100,0.74\end{array}$ & & $\begin{aligned} \mathrm{ATU} & =0.012-0.042 \\
K_{G} a & =340 \mathrm{~mol} / \mathrm{m}^{3} \mathrm{~s}\end{aligned}$ & \\
\hline $\begin{array}{l}\text { Acetone/water } \\
\text { (TSCC-RPB/ cont. distill.) }\end{array}$ & $\begin{array}{l}(0.0725 \\
0.178,0.046)\end{array}$ & - & $\begin{array}{l}\text { SS WM, } \\
670,0.96\end{array}$ & $\begin{array}{l}400- \\
1200\end{array}$ & $\mathrm{HETP}=1.73-4.05$ & $\begin{array}{l}\text { (Luo et al., } \\
2012)^{30}\end{array}$ \\
\hline $\begin{array}{l}\text { Methanol/Ethanol } \\
\text { (SP-RPB/ Tot. reflux) }\end{array}$ & $\begin{array}{l}(0.03,0.155 \\
0.027)\end{array}$ & - & $\begin{array}{l}\text { SS WM, } \\
280,-\end{array}$ & $\begin{array}{l}600- \\
1550\end{array}$ & $\begin{array}{l}\text { HETP }=2.9-15.0 \\
\text { ATU }=0.02-0.1 \mathrm{~m}^{2}\end{array}$ & $\begin{array}{l}\text { (Mondal et } \\
\text { al., 2012) }\end{array}$ \\
\hline
\end{tabular}


Methanol/water

(TSCC-RPB/ cont. distill.)

Ethanol/water

(CFCR-RB/ Tot. reflux)

Ethanol/water

(CRB/ Tot. reflux)

Methanol/water

(TSBP/ cont. distill.)

\section{$(0.0725$ \\ $0.183,0.058)$ \\ $10 \mathrm{~L} / \mathrm{h}$ \\ SS WM \\ $670,0.96$ \\ Porous \\ sheet: $4 \mathrm{~mm}$ \\ thickness \\ Packing \\ ring: $16 \mathrm{~mm}$ \\ thickness}

400

HETP=3.12 - 4.72

1000

Chu et al. 2013) ${ }^{31}$

(0.07, 0.136,

$(0.015$,

0.045))

Concentric

$800-$

1400

HETP=3.0 - 6.5

$K_{G} a=3.18-92.30 \mathrm{~s}^{-1}$

$K_{G}=0.1-0.6 \mathrm{~m} / \mathrm{s}$

$k_{G}=0.1-1.1 \mathrm{~m} / \mathrm{s}$

$k_{L}=0.001-0.002 \mathrm{~m} / \mathrm{s}$

Concentric $\quad 400-\quad$ Stage efficiency $10-15 \%$

baffles,-,- 1200

0.08)

$(0.064,0.174, \quad 20-140$

0.040 )

$\mathrm{L} / \mathrm{h}, 1600$

- 8000L/h
HETP $=1.9-10.0$

$k_{L}=0.0005-0.0019 \mathrm{~m} / \mathrm{s}$

$a=93-337 \mathrm{~m}^{2} / \mathrm{m}^{3}$
(Li et al., $2014)^{48}$

(Wang et al., $2014)^{49}$

(Luo et al., $2016)^{47}$
$600-$

rings (SS 1400

WM, 500

$\left.\mathrm{m}^{2} / \mathrm{m}^{3}, 0.96\right)$

and blade

rings. 
1 Table 3. Overview of works published on RPB modeling with a first-principles approach

\begin{tabular}{|c|c|c|c|c|c|}
\hline Application & RPB config. & Model description & $\begin{array}{c}\text { G-L Mass } \\
\text { transfer } \\
\text { model }\end{array}$ & $\begin{array}{l}\text { Liquid } \\
\text { flow } \\
\text { (assumed) }\end{array}$ & References \\
\hline $\begin{array}{l}\text { Chemical absorption of } \mathrm{CO} 2 \\
\text { in } \mathrm{NaOH} \text { and dissolution of } \\
\text { naphthalene in water }\end{array}$ & Counter-flow & $\begin{array}{l}\text { Gas-liquid and } \\
\text { liquid-solid mass } \\
\text { transfer }\end{array}$ & $\begin{array}{l}\text { Penetration } \\
\text { theory and } \\
\text { convection- } \\
\text { diffusion } \\
\text { model }\end{array}$ & Film flow & $\begin{array}{l}\text { Munjal et } \\
\mathrm{al}^{82,123}\end{array}$ \\
\hline $\begin{array}{l}\text { Selective } \mathrm{H}_{2} \mathrm{~S} \text { absorption in } \\
\text { MDEA }\end{array}$ & Counter-flow & $\begin{array}{l}\text { Reaction- } \\
\text { equilibrium-mass } \\
\text { transfer }\end{array}$ & $\begin{array}{l}\text { Penetration } \\
\text { theory }\end{array}$ & Film flow & $\begin{array}{l}\text { Quian et } \\
\text { al. }^{89,90}\end{array}$ \\
\hline $\mathrm{CO}_{2}$ absorption in MDEA & Counter-flow & $\begin{array}{l}\text { Diffusion-reaction } \\
\text { mass-transfer }\end{array}$ & $\begin{array}{l}\text { Penetration } \\
\text { theory }\end{array}$ & Film flow & $\begin{array}{l}\text { Zhang et } \\
\text { al. }{ }^{144}\end{array}$ \\
\hline $\begin{array}{l}\text { Absorption of } \mathrm{SO}_{2} \text { into } \\
\text { aqueous } \mathrm{NaOH} \text { and stripping } \\
\text { of } \mathrm{O}_{2} \text { from water }\end{array}$ & Counter-flow & $\begin{array}{l}\text { Mass transfer } \\
\text { coefficients }\end{array}$ & $\begin{array}{l}\text { Two-film } \\
\text { theory }\end{array}$ & $N / A$ & $\begin{array}{l}\text { Shivhare et } \\
\text { al. }^{39}\end{array}$ \\
\hline $\begin{array}{l}\mathrm{CO}_{2} \text { absorption by Benfield } \\
\text { solution }\end{array}$ & Counter-flow & $\begin{array}{l}\text { Gas-liquid mass } \\
\text { transfer with } \\
\text { reaction }\end{array}$ & $\begin{array}{l}\text { Two-film } \\
\text { theory }\end{array}$ & $\begin{array}{l}\text { Droplet } \\
\text { flow }\end{array}$ & Yi et al. ${ }^{86}$ \\
\hline $\begin{array}{l}\text { Simultaneous absorption of } \\
\mathrm{CO}_{2} \text { and } \mathrm{NH}_{3} \text { into water }\end{array}$ & Counter-flow & $\begin{array}{l}\text { Gas-liquid mass } \\
\text { transfer }\end{array}$ & $\begin{array}{l}\text { Two-film } \\
\text { theory }\end{array}$ & $\begin{array}{l}\text { Film and } \\
\text { droplet } \\
\text { flow }\end{array}$ & Sun et al. ${ }^{85}$ \\
\hline $\begin{array}{l}\text { Ozonation of pollutant (o- } \\
\text { Cresol) }\end{array}$ & Counter-flow & $\begin{array}{l}\text { Gas-liquid mass } \\
\text { transfer with } \\
\text { reaction }\end{array}$ & $\begin{array}{l}\text { Two-film } \\
\text { theory }\end{array}$ & Film flow & Chen et al. ${ }^{84}$ \\
\hline $\begin{array}{l}\text { Absorption of volatile organic } \\
\text { compounds (VOCs) into } \\
\text { water }\end{array}$ & Cross-flow & $\begin{array}{l}\text { Gas-liquid mass } \\
\text { transfer }\end{array}$ & $\begin{array}{l}\text { Surface } \\
\text { renewal } \\
\text { theory }\end{array}$ & $\begin{array}{l}\text { Film and } \\
\text { droplet } \\
\text { flow }\end{array}$ & Chen et al. ${ }^{55}$ \\
\hline $\begin{array}{l}\text { Water deaeration, } \mathrm{NH}_{3} \\
\text { absorption and } \mathrm{SO}_{2} \text { chemical } \\
\text { absorption }\end{array}$ & Cross-flow & $\begin{array}{l}\text { Gas-liquid mass } \\
\text { transfer (reaction) }\end{array}$ & $\begin{array}{l}\text { Surface } \\
\text { renewal } \\
\text { theory }\end{array}$ & $\begin{array}{l}\text { Film and } \\
\text { droplet } \\
\text { flow }\end{array}$ & Guo et al. ${ }^{56}$ \\
\hline $\begin{array}{l}\text { Solid catalyzed reactive } \\
\text { stripping }\end{array}$ & Counter-flow & $\begin{array}{l}\text { Gas-liquid mas } \\
\text { transfer with solid } \\
\text { catalyzed reaction }\end{array}$ & $\begin{array}{l}\text { Two-film } \\
\text { theory }\end{array}$ & Film flow & $\begin{array}{l}\text { Gudena et } \\
\text { al. }^{79}\end{array}$ \\
\hline
\end{tabular}


2 Table 4. List of major industrial applications of HiGee gas-liquid contactors reported in the 3 literature

\begin{tabular}{|c|c|c|c|c|c|}
\hline Company & Application & Capacity & Year & Remarks & Ref. \\
\hline $\begin{array}{l}\text { China Petrochemical } \\
\text { Corporation/ Shengli } \\
\text { Oil Field Co., China }\end{array}$ & $\begin{array}{l}\text { Seawater } \\
\text { deaeration }\end{array}$ & $\begin{array}{l}\text { Two units } \\
\text { of } 250 \mathrm{t} / \mathrm{h}\end{array}$ & 1998 & $\begin{array}{l}\text { - Investment cost } 40 \% \text { lower. } \\
\text { - Ground space reduced by } 60 \% \text {. } \\
\text { - Equipment weight reduced by } 80 \% \text {. } \\
\text { - Improved oxygen removal efficiency. }\end{array}$ & 105 \\
\hline $\begin{array}{l}\text { The Dow Chemical } \\
\text { Company, USA }\end{array}$ & $\begin{array}{l}\text { Reactive } \\
\text { stripping of } \\
\mathrm{HOCl}\end{array}$ & $150 \mathrm{t} / \mathrm{h}$ & 1999 & $\begin{array}{l}\text { - Yield } 10 \% \text { higher. } \\
\text { - Less than half stripping gas. } \\
\text { - Investment cost } 70 \% \text { lower. } \\
\text { - Operating cost } 30 \% \text { lower. } \\
\text { - Reactor volume } 40 \% \text { smaller. }\end{array}$ & $\begin{array}{l}32,106,1 \\
45\end{array}$ \\
\hline $\begin{array}{l}\text { Zibo Sulphuric acid } \\
\text { plant (Shandong } \\
\text { province, P. R. } \\
\text { China), }\end{array}$ & $\begin{array}{l}\mathrm{SO}_{2} \\
\text { absorption } \\
\text { from tail gas } \\
\text { of } \mathrm{SO}_{3} \\
\text { absorber. }\end{array}$ & $\begin{array}{l}3000 \mathrm{~m}^{3} / \mathrm{h} \\
\text { of gas }\end{array}$ & 1999 & $\begin{array}{l}\text { - Degree of equilibrium absorption } \\
\text { close to } 100 \% \text {. } \\
\text { - Compared with conventional tower, } \\
\text { capital investment reduced by } 35 \% \text {. } \\
\text { - Volume reduced by } 50 \% \text {. } \\
\text { - Energy consumption reduced by } \\
25 \% \text {. }\end{array}$ & $\begin{array}{l}26,146 \\
147\end{array}$ \\
\hline $\begin{array}{l}\text { Fujian Petroleum } \\
\text { Refinery Co., China }\end{array}$ & $\begin{array}{l}\mathrm{H}_{2} \mathrm{~S} / \mathrm{CO}_{2} \\
\text { selective } \\
\text { absorption in } \\
\text { MDEA }\end{array}$ & $\begin{array}{l}11 \mathrm{t} / \mathrm{h} \\
\text { (feed gas) }\end{array}$ & & $\begin{array}{l}\text { - } \mathrm{CO}_{2} \text { co-absorption reduced from } \\
79.9 \% \text { to } 8.9 \% \text {. } \\
\text { - Equipment volume reduced from } 36.1 \\
\mathrm{~m}^{3} \text { to } 3.4 \mathrm{~m}^{3} \text {. } \\
\text { - Packing volume reduced from } 14 \mathrm{~m}^{3} \\
\text { to } 0.3 \mathrm{~m}^{3} \text {. } \\
\text { - Steady state reached in few minutes. } \\
\text { - Lower power requirement than } \\
\text { packed bed. }\end{array}$ & 89,105 \\
\hline $\begin{array}{l}\text { PepsiAmericas Plant, } \\
\text { USA }\end{array}$ & $\begin{array}{l}\text { Water } \\
\text { deaeration } \\
\text { for soft drink } \\
\text { bottling }\end{array}$ & $\mathrm{N} / \mathrm{A}$ & $\begin{array}{l}2006 \\
2008\end{array}$ & $\begin{array}{l}\text { - Lower dissolved oxygen and } \mathrm{CO}_{2} \\
\text { levels (from } 8.1 \mathrm{ppm} \text { to } 0.3-0.6 \mathrm{ppm} \text {, } \\
\text { at } 9-6^{\circ} \mathrm{C} \text { ) without stripping gases. } \\
\text { - Annual savings (Indianapolis plant) of } \\
\text { ( } \$ 88,000 \text { ). } \\
\text { - Filling speeds increased by } 10 \%-40 \% \\
\text { - Improvement in the distribution of } \\
\text { carbonation levels. } \\
\text { - Increased fill accuracy. }\end{array}$ & 110,148 \\
\hline
\end{tabular}


1 Table 5. List of suppliers of HiGee equipment.

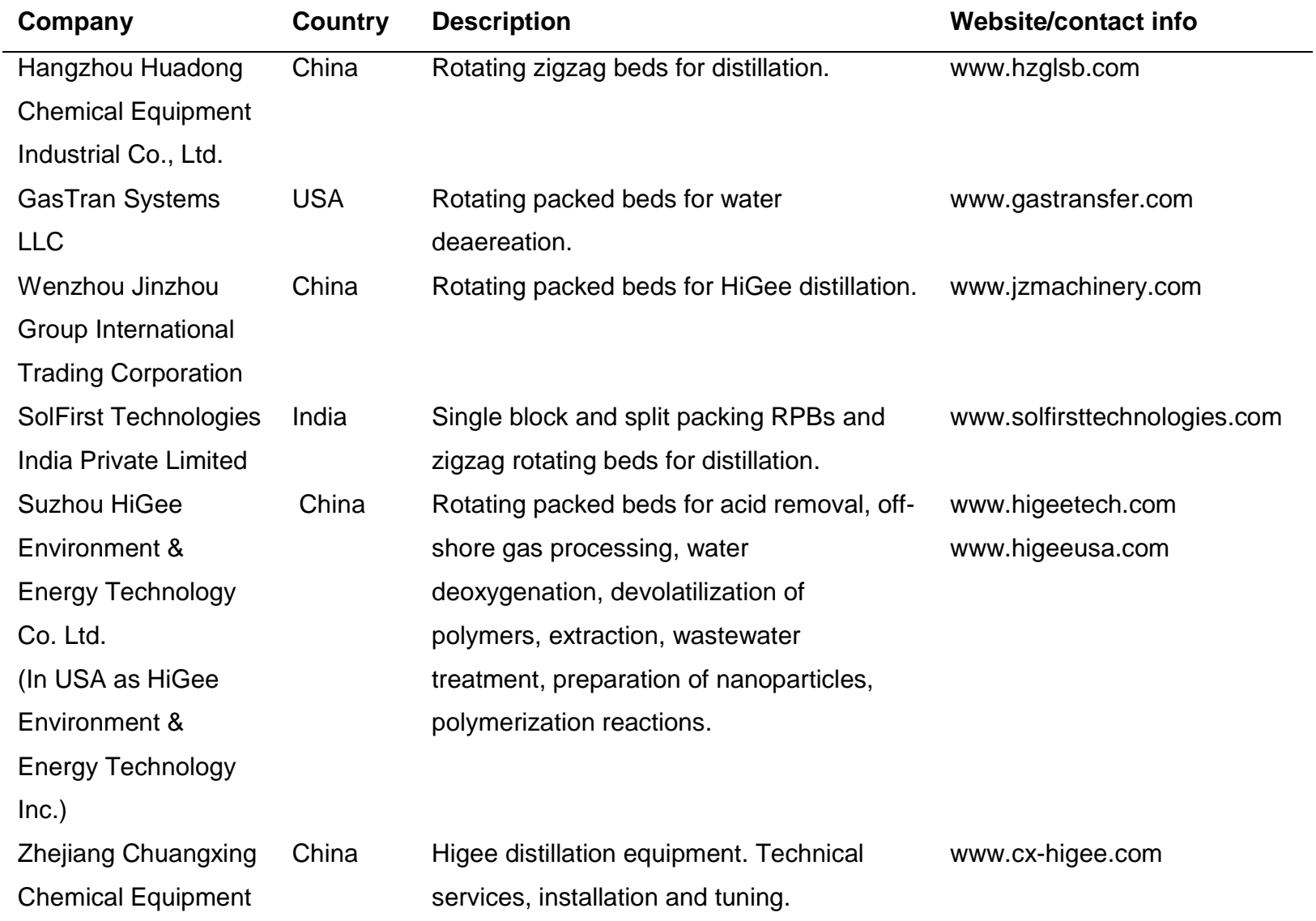

Co., Ltd 


\section{$1 \quad$ Figure captions}

2

3 4

Figure 12. Design analogy between conventional and HiGee distillation

Figure 1. Working principle of HiGee distillation in a rotating packed bed (RPB) flow RPB, (btm) cross-current flow

Figure 3. Schematic drawing of a split-packing rotating packed-bed

Figure 4. Schematic drawing of the rotor of the RZB

Figure 5. Schematic drawing of the rotor (one stage) of a TSCC-RPB

Figure 6. Arrangement of blade packings in RPB structure of the RPB packings and baffles.

Figure 9. Schematic drawing of a counter-flow concentric-ring rotating bed

Figure 10. Schematic drawing of a crossflow concentric-baffle rotating bed (CRB) vapor flow, dashed arrows: liquid flow

Figure 2. Schematic drawing of a single-block rotating packed bed: (top) Counter-current

Figure 7. Schematic drawing of RPB with packing and blades (top) Rotor design; (btm) main

Figure 8. Schematic drawing of blades and baffles rotating bed; (left) blade packings on the rotational disk and baffles on the stationary disk; (right) structure of the RPB with blade

Figure 11. Schematic drawing showing the method followed by Gudena et al.95 to approximate HiGee (left sketch) as a conventional column (right sketch). Continuous arrows: 


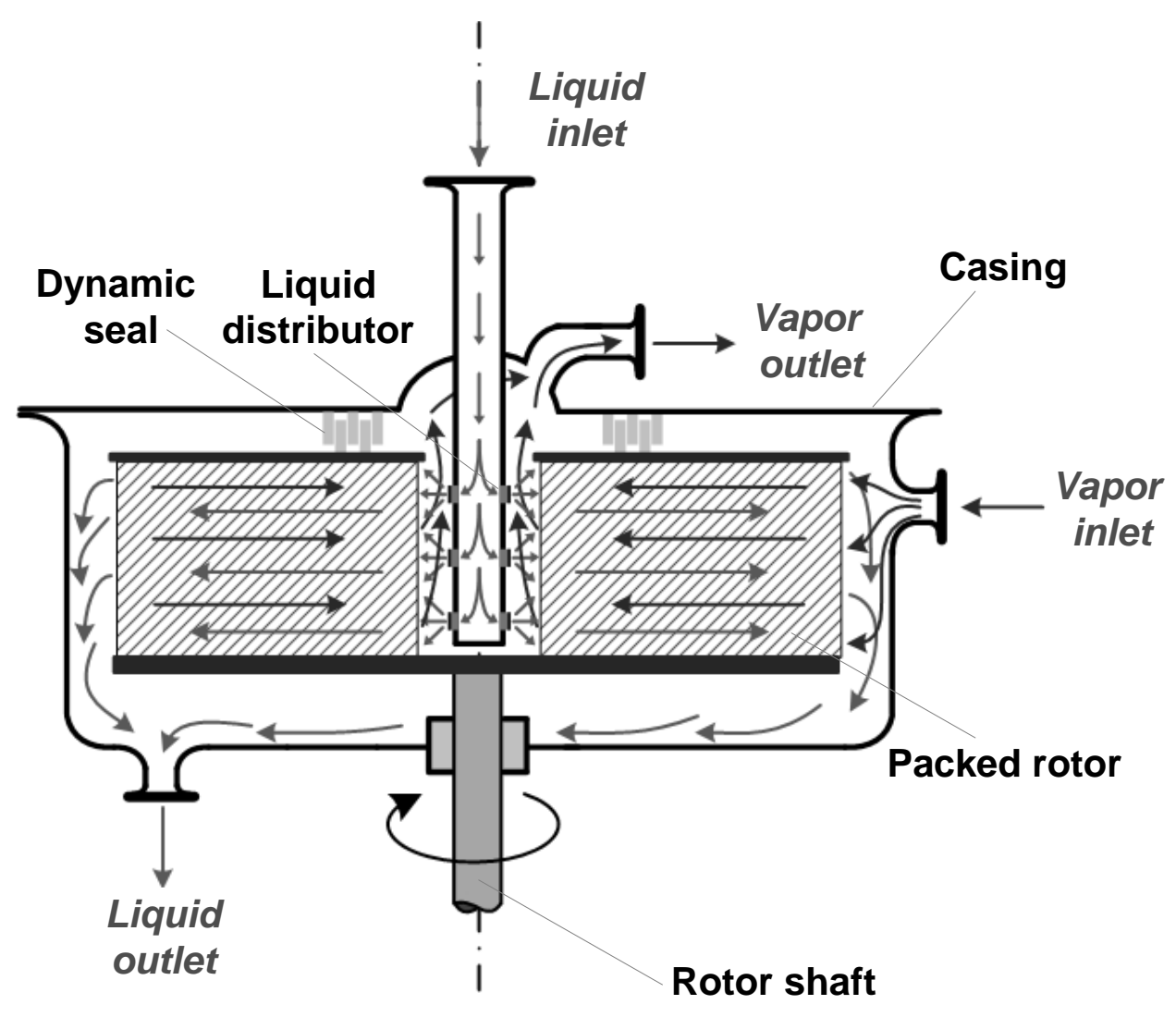



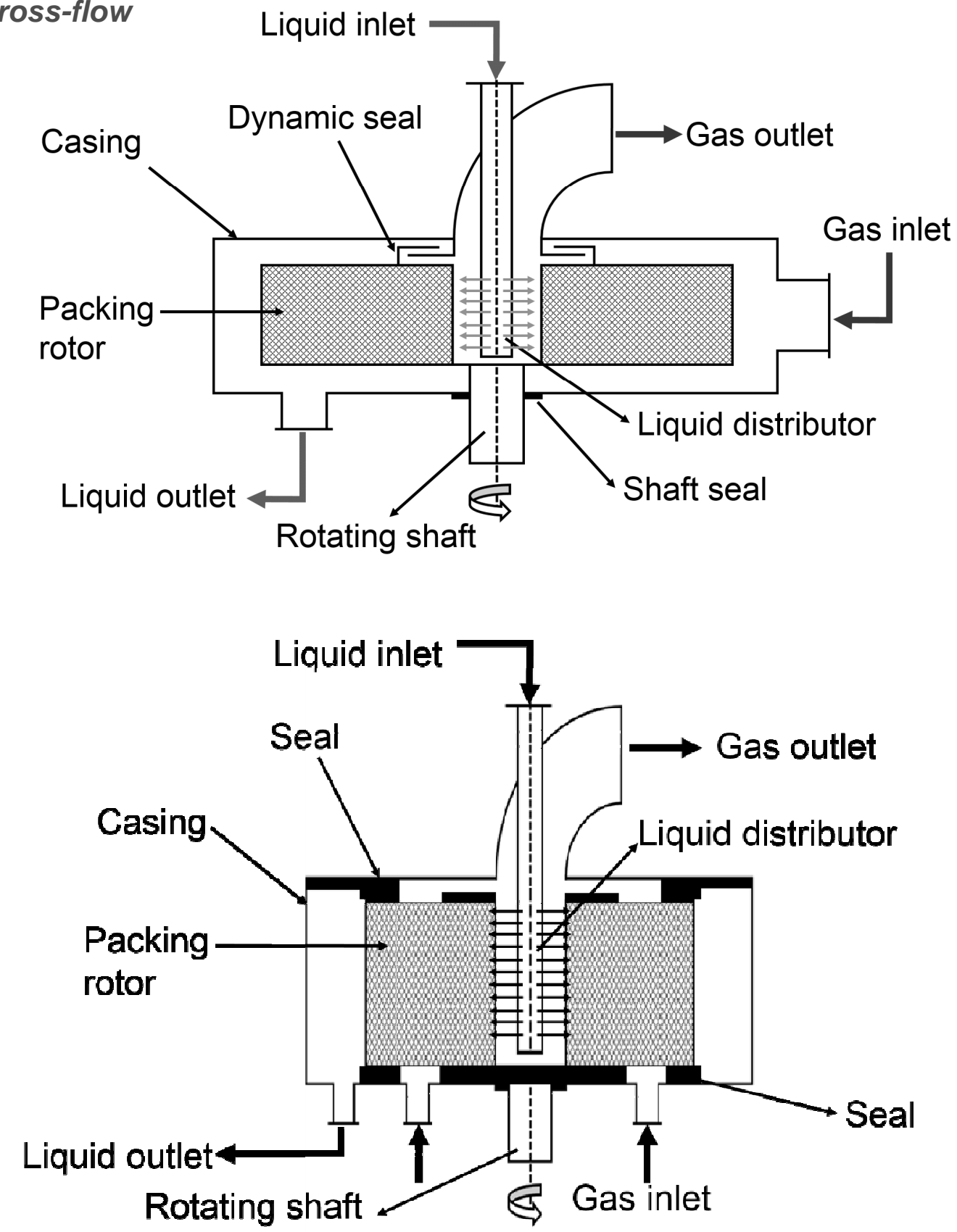


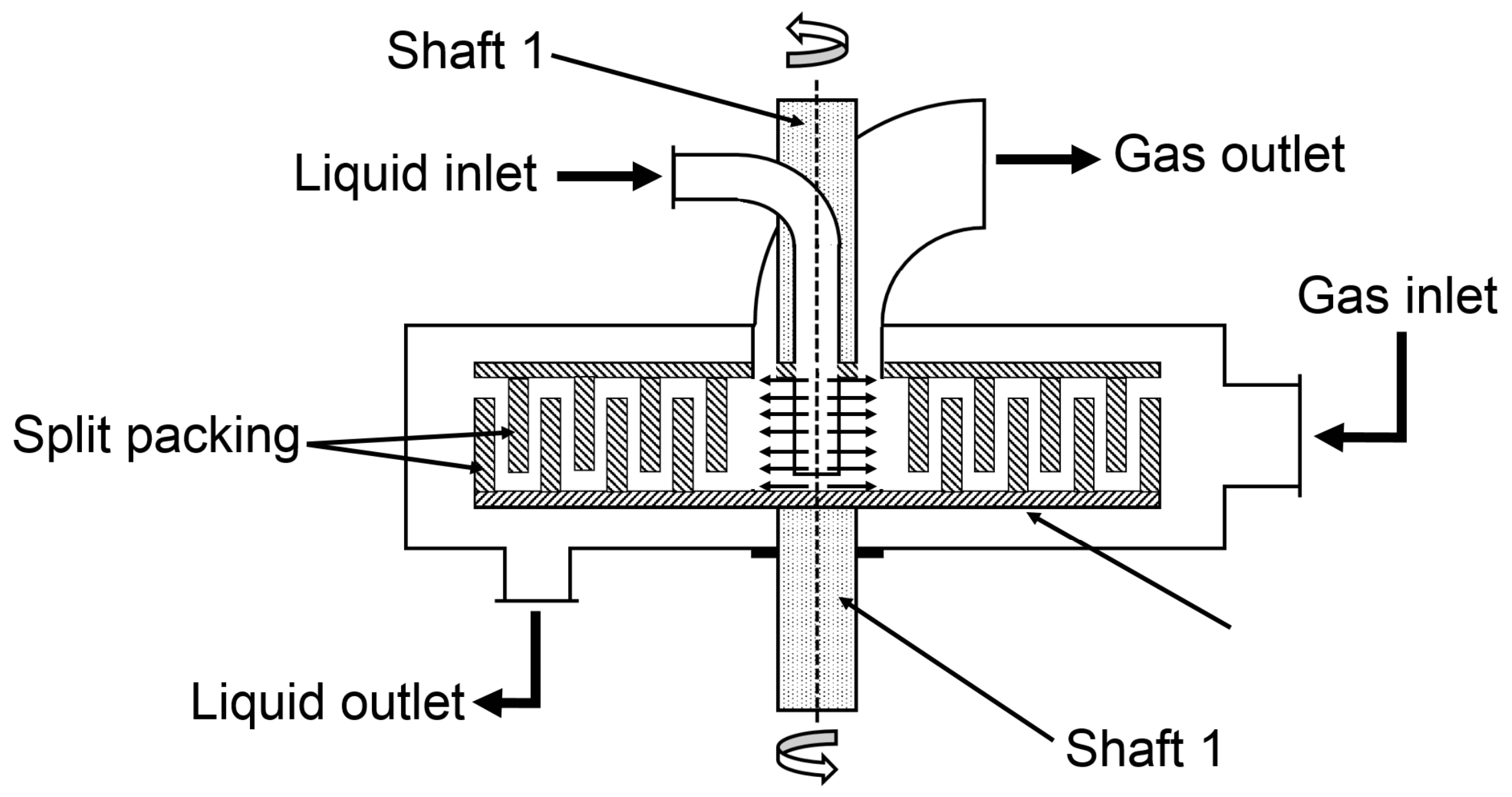




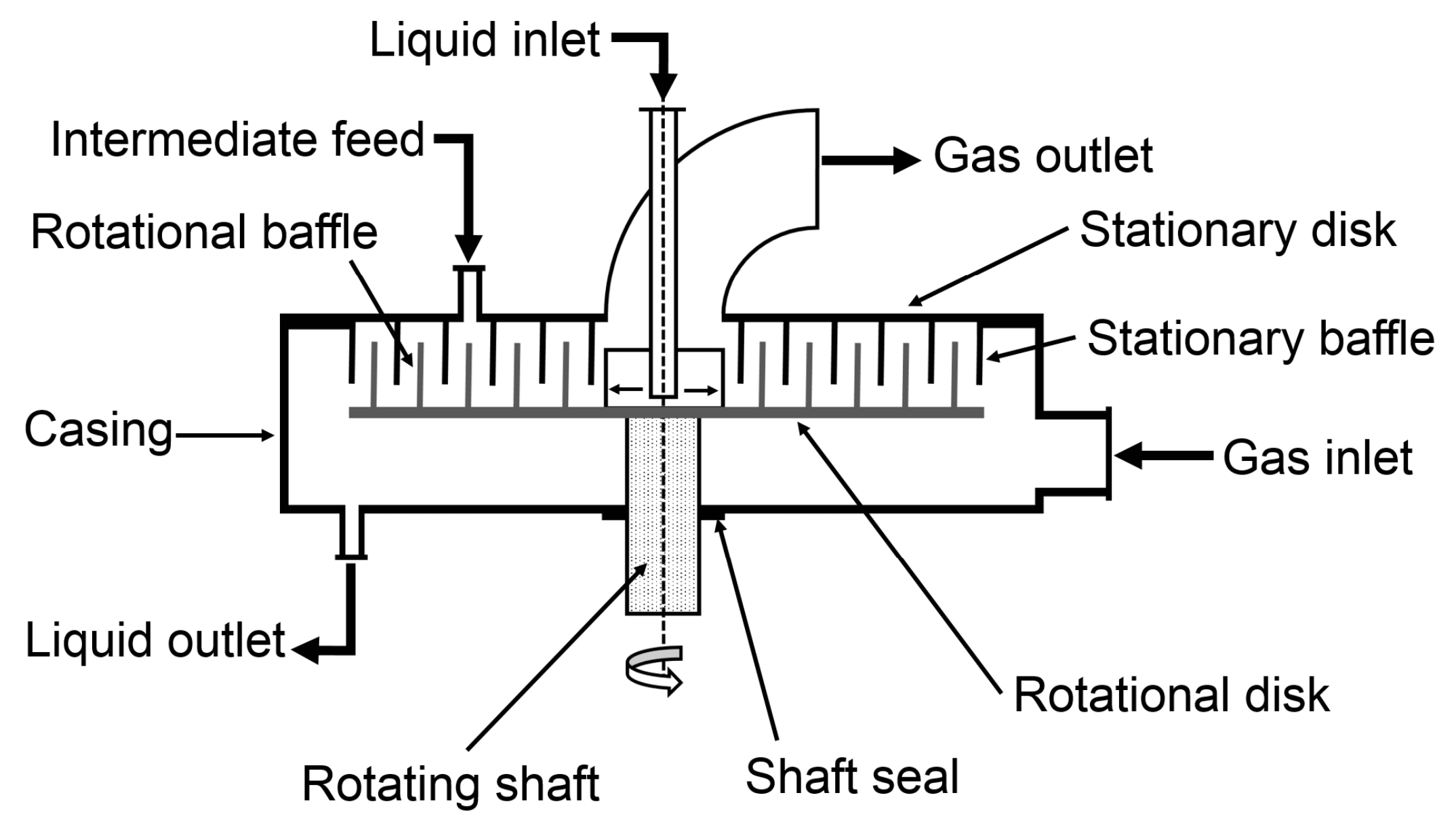




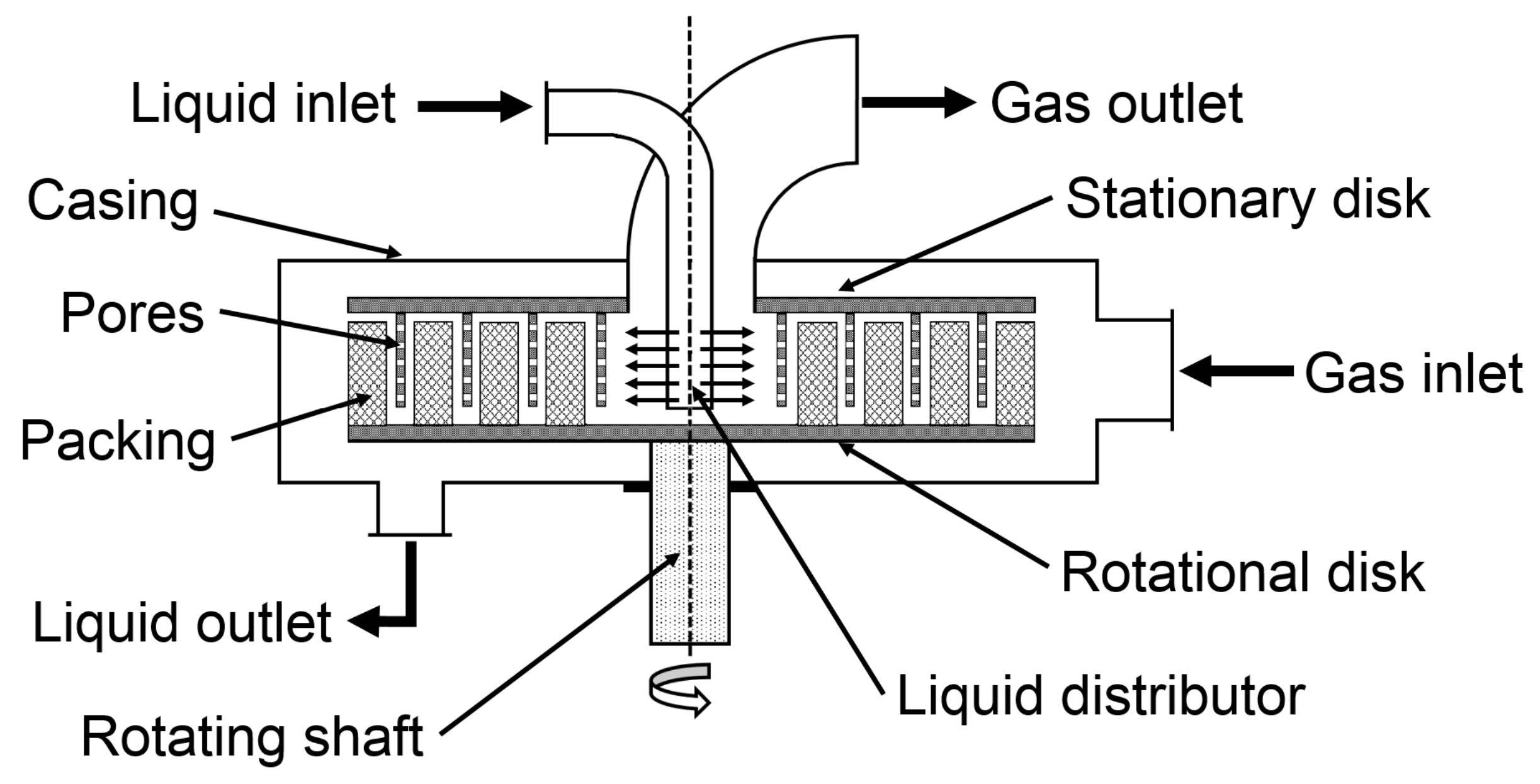




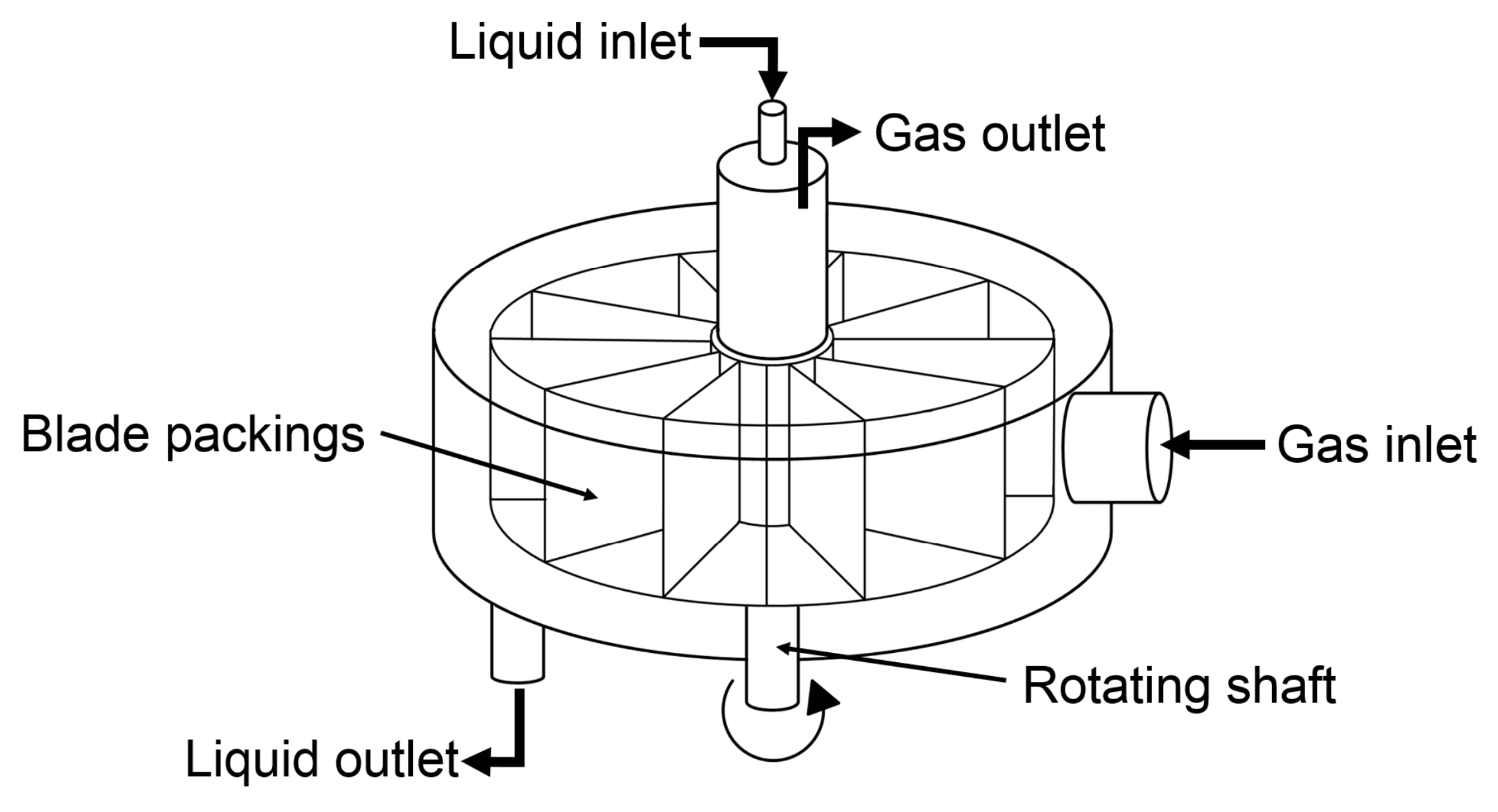



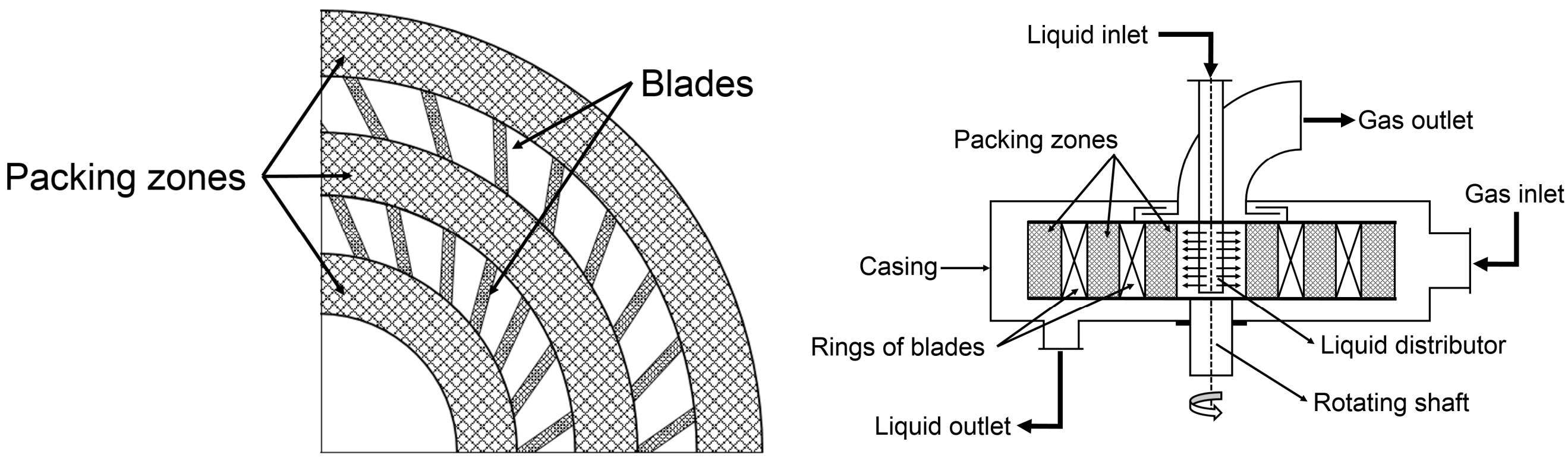
(left) blade packings on the rotational disk and baffles on the stationary disk (right) structure of the RPB with blade packings and baffles
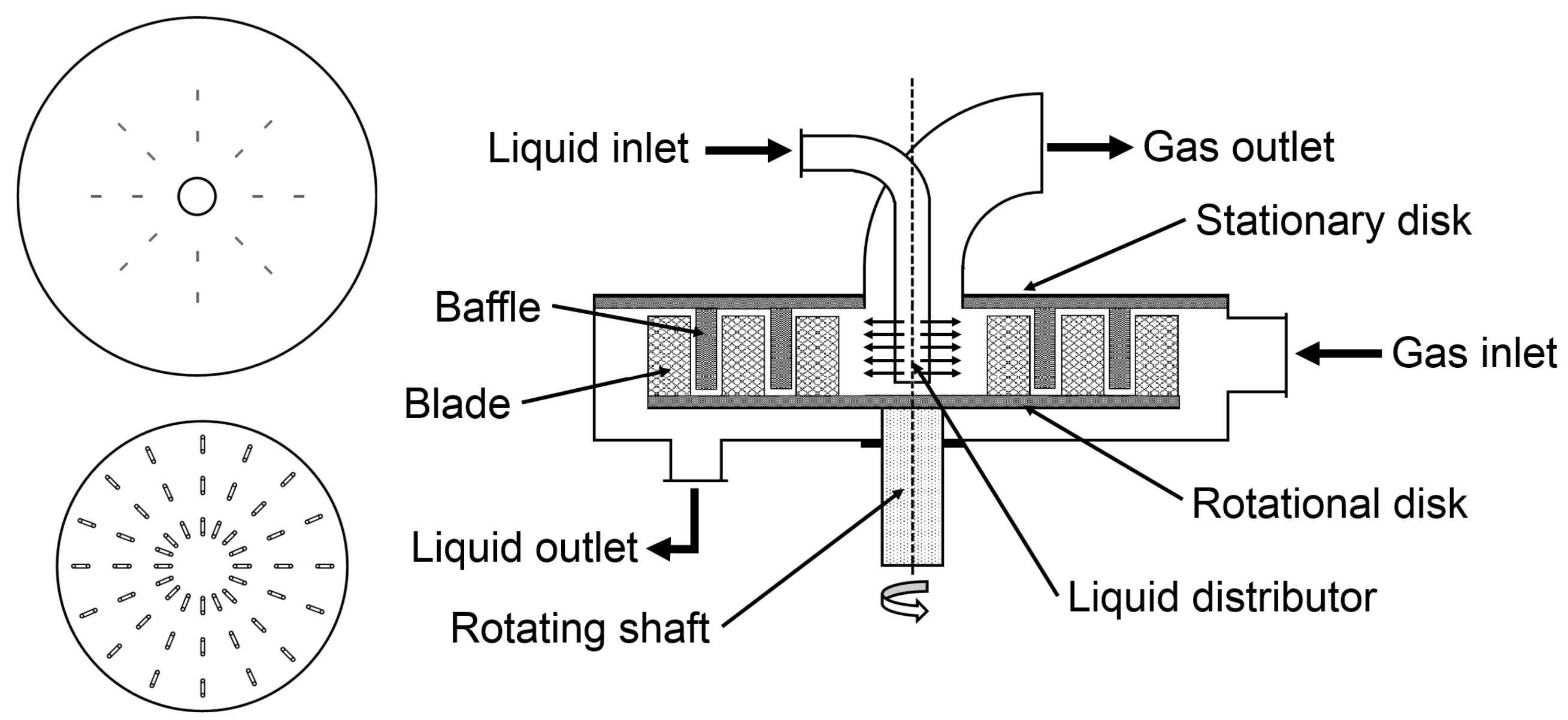


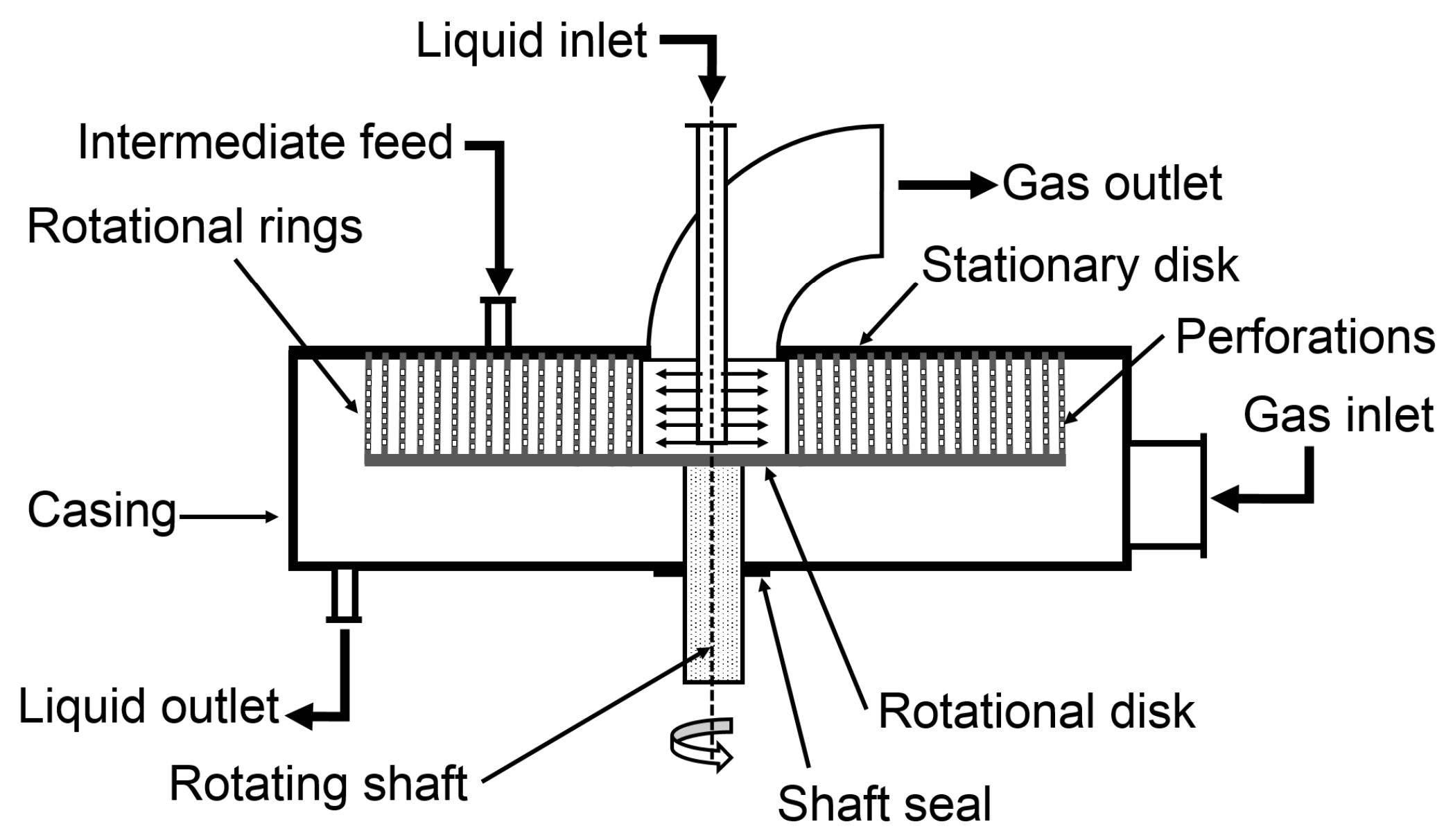




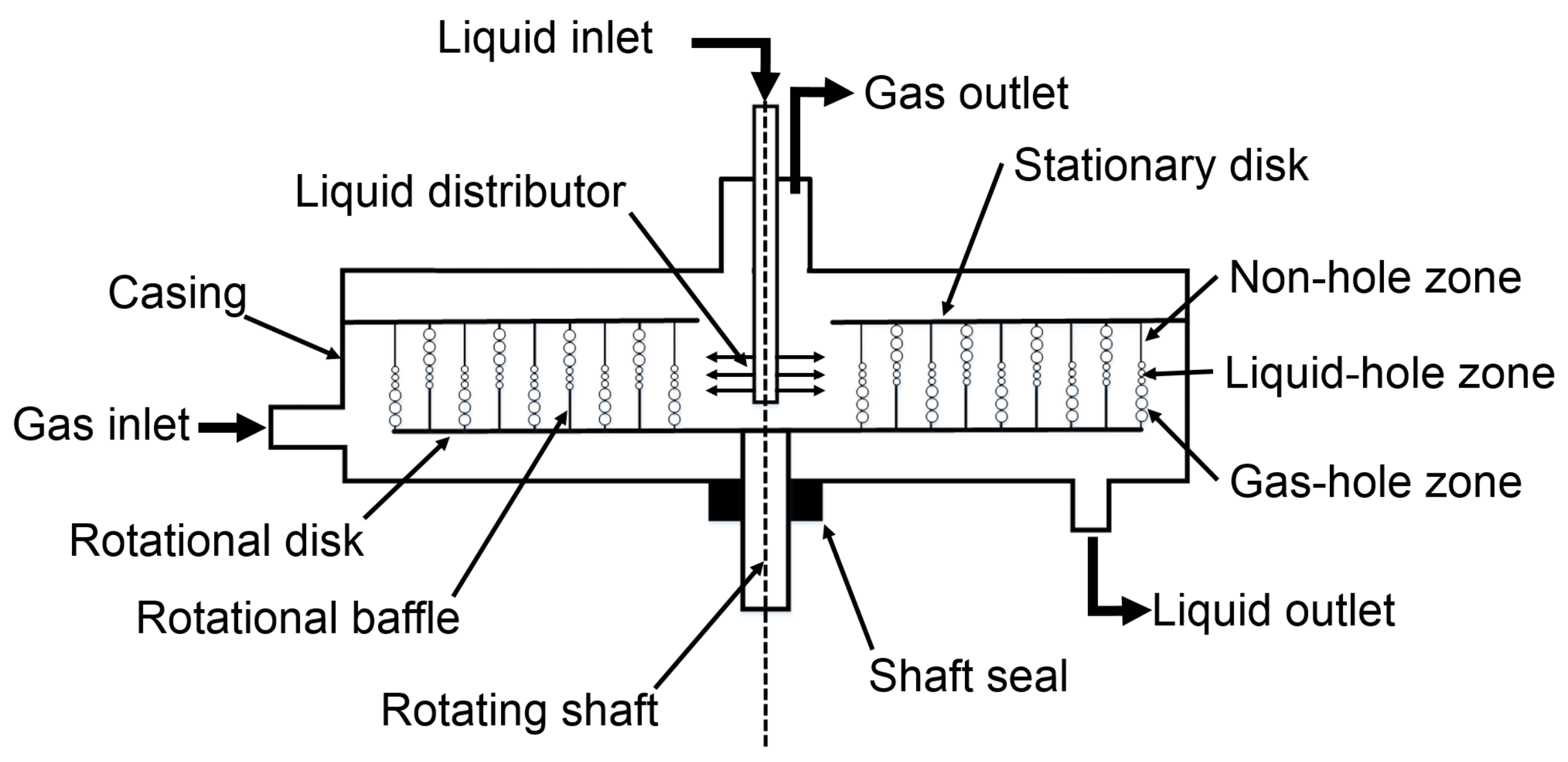



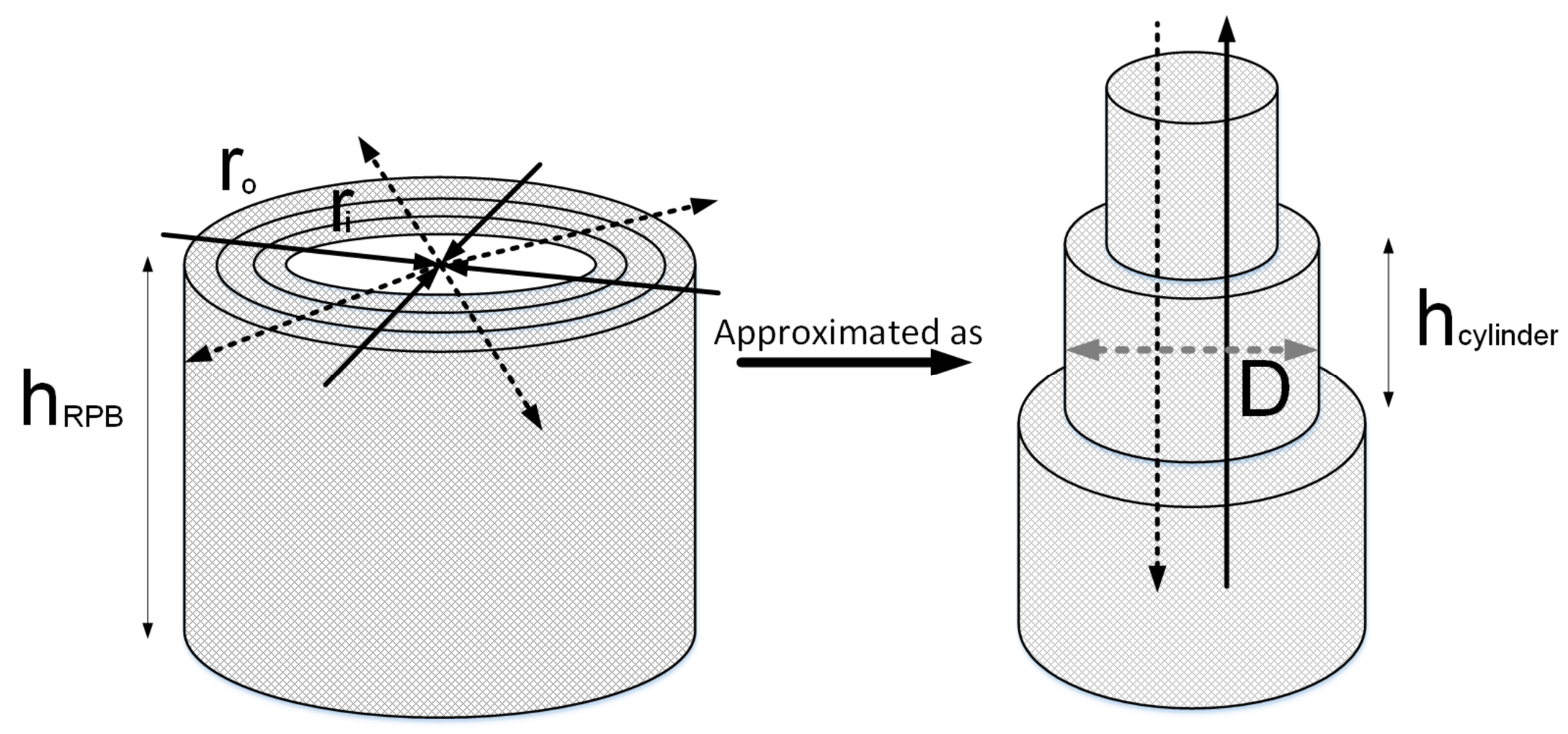


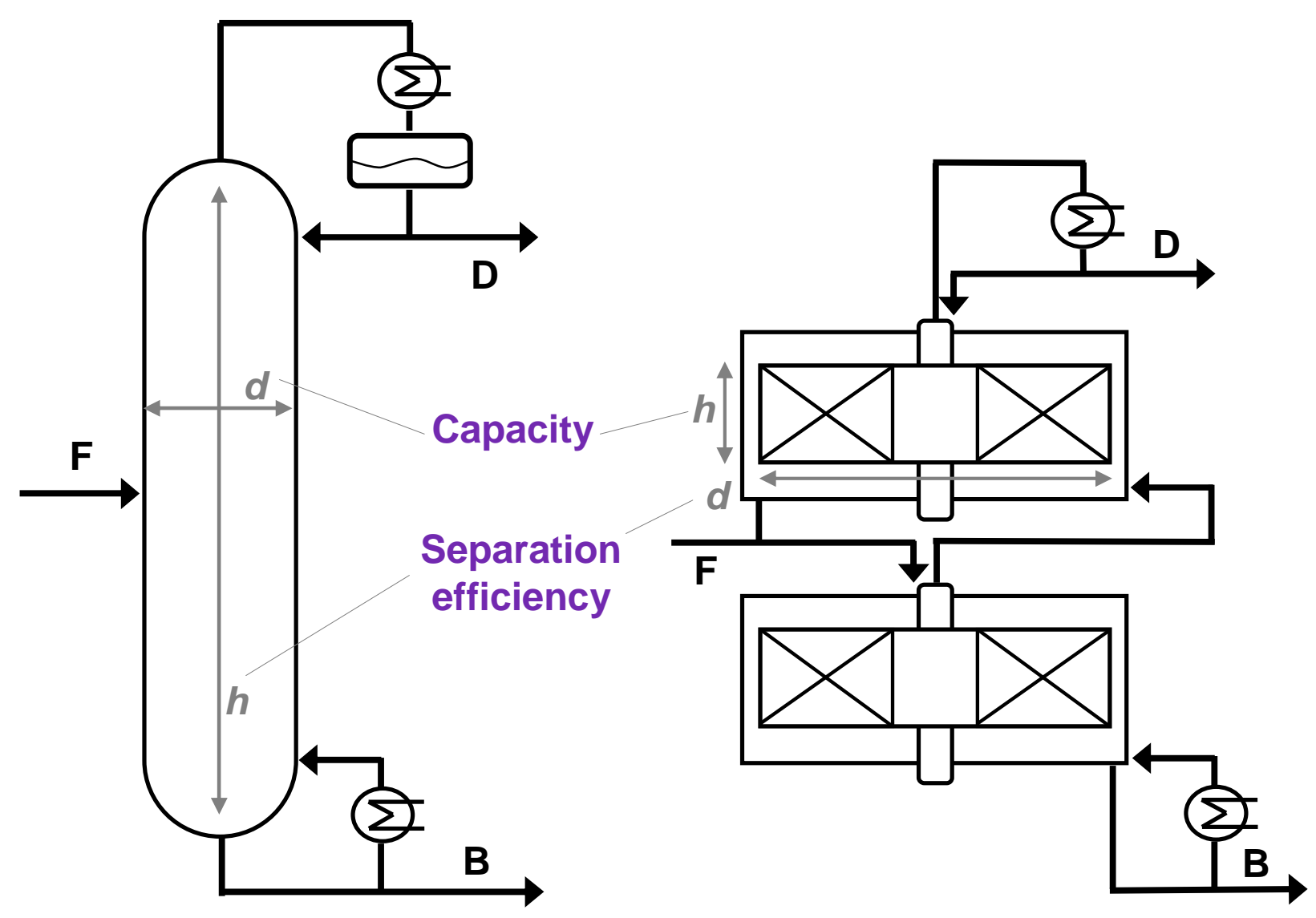

